\title{
The shape of things to come: why is climate change so predictable?
}

\author{
Marcia B. Baker and Gerard H. Roe \\ Department of Earth and Space Sciences, \\ University of Washington, Seattle, WA.
}

February 1, 2009 


\begin{abstract}
The framework of feedback analysis is used to explore the controls on the shape of the probability distribution of global mean surface temperature response to climate forcing. It is shown that ocean heat uptake, which delays and damps the temperature rise, can be represented as a transient negative feedback. This transient negative feedback causes the transient climate change to have a narrower probability distribution than that of the equilibrium climate response (the climate sensitivity). In this sense, climate change is much more predictable than climate sensitivity. The width of the distribution grows gradually over time, a consequence of which is that the larger the climate change being contemplated, the greater the uncertainty is about when that change will be realized. Another consequence of this slow growth is that further efforts to constrain climate sensitivity will be of very limited value for climate projections on societally-relevant time scales. Finally, it is demonstrated that the effect on climate predictability of reducing uncertainty in the atmospheric feedbacks is greater than the effect of reducing uncertainty in ocean feedbacks by the same proportion. However, at least at the global scale, the total impact of uncertainty in climate feedbacks is dwarfed by the impact of uncertainty in climate forcing, which in turn is contingent on choices made about future anthropogenic emissions.
\end{abstract}




\section{Introduction}

Projections of future climate changes are inherently probabilistic, because of the many uncertainties surrounding choices of climate parameters, uncertainties in the underlying physical processes and future emissions, and because the climate system is highly complex. On the other hand, such projections are now recognized as essential tools for planners at all levels in our society, as the global temperature rises and approaches levels which cannot be reversed in the next few centuries. While the projections are usually made on the basis of highly sophisticated and expensive numerical models, we present here a much simpler set of tools that provides understanding and estimation of uncertainties in these projections.

Climate sensitivity is defined as the equilibrium response of the global- and annual-mean surface air temperature to a doubling of carbon dioxide over pre-industrial values (e.g., IPCC, 2007, hereafter IPCC07), and has been a standard metric via which to compare different estimates of climate change ever since the initial estimates of Arrhenius (1896). From a climate modeling perspective, determining the envelope of uncertainty in climate sensitivity requires understanding how uncertainty in the representations of physical processes in global climate models (GCMs) translates into the uncertainty in the models' response. Roe and Baker (2007) (hereafter, RB07) investigated this question using the framework of feedback analysis (e.g., Maxwell, 1886; Cess, 1976; Hansen et al., 1984; Schlesinger and Mitchell, 1985; Roe, 2008). The net effect of atmospheric feedbacks is that the climate system acts as a strong amplifier of external radiative forcing. RB07 showed that one consequence of this amplification is that a symmetric distribution of uncertainty in feedbacks leads to a strongly skewed distribution of uncertainty in climate sensitivity. For a step increase $\Delta R_{F}=$ constant in radiative forcing, and a Gaussian-shaped distribution of uncertainty in feedbacks characterized by a mean $\bar{f}$ and standard deviation $\sigma_{f}$, RB07 showed the equilibrium 
probability density function (PDF) of the climate sensitivity, or global mean surface temperature $T$, is given by

$$
h_{T}(T, \infty)=\left(\frac{1}{\sigma_{f} \sqrt{2 \pi}}\right) \frac{T_{0}}{T^{2}} \exp \left[-\frac{1}{2}\left(\frac{\left(1-\bar{f}-\frac{T_{0}}{T}\right)}{\sigma_{f}}\right)^{2}\right],
$$

where $T_{0} \equiv \lambda_{0} \Delta R_{F} ; \lambda_{0} \approx 0.3 \mathrm{~K}\left(\mathrm{~W} \mathrm{~m}^{-2}\right)^{-1}$ (e.g., Hansen et al., 1984).

$h(T, \infty)$ is shown as the dark red curve in Figure 1 for the Gaussian distribution of feedbacks shown in dark green $\left(\bar{f}=0.65 ; \sigma_{f}=0.13\right)$. This shape is consistent with results from multi-ensemble GCM studies as well as a variety of observationally-based estimates. The lighter curves, to be discussed later, show the same distributions for $\bar{f}=0.5, \sigma_{f}=0.13$.

RB07 also showed that for the larger $\bar{f}$ the strong skewing of the probability distribution towards high climate sensitivities means that estimates of the likelihood of climate sensitivity exceeding the central range of the IPCC07 estimate of 2 to $4.5{ }^{\circ} \mathrm{C}$ are not sensitive to the uncertainty in the climate feedbacks. In other words, reducing uncertainty in model parameterizations do not help very much in constraining the fat tail of high climate sensitivity estimates.

By definition, climate sensitivity is a measure of equilibrium climate change. As several studies have shown, the adjustment of the deep ocean actually takes several centuries or more to come into equilibrium (e.g., Hoffert, 1980; Hansen et al., 1985; Wigley and Schlesinger, 1985; Allen and Frame, 2007). This timescale is only weakly constrained because the processes of ocean adjustment remain poorly known. Importantly though, the higher the climate sensitivity, the longer the adjustment time and so the fat tail of the PDF of climate response grows only slowly with time (e.g., Hansen et al., 1985; Wigley and Schlesinger, 1985). As pointed out by Frame et al. (2006) and Allen and Frame (2007), this long adjustment time calls into question the value of better constraining climate sensitivity in making climate projections that inform climate policy choices over the coming 
decades and centuries.

In this study we extend the investigation of RB07 to the time dependent evolution of the PDF of the planetary mean surface temperature under the action of anthropogenic radiative forcing. Our goal is to identify general principles governing the evolving shape of the function $h_{T}(T, t)$, which we define as the probability density that the global mean surface temperature anomaly has value $T$ at time $t$ after the initiation of the radiative forcing. We demonstrate that, as in the case of equilibrium climate sensitivity, the transient response of the system can be cleanly characterized in terms of climate feedbacks. The uptake of heat by the deep ocean can be interpreted as a transient negative feedback. With this perspective, the central questions of RB07 can be addressed for the transient case. Firstly, how does the uncertainty in climate feedbacks translate into uncertainty in the transient climate reponse? Secondly, how does a reduction of uncertainty in model parameters (or feedbacks) translate into uncertainty in the system response $T(t)$ ? Our work complements previous investigations of uncertainty in climate predictions (e.g., Wigley and Raper, 2001; Frame et al., 2005; Knutti et al., 2005) in that we focus on general and qualitative features of the probability distributions of predicted parameters, rather than on optimizing a quantitative constraint on their values.

In order to make projections of global mean surface temperature over the next few centuries, we must account for heat buried in the oceans. Imposition of net radiative forcing from above warms the upper ocean, which loses heat to the vast ocean below. This heat loss reduces the rate of warming of the ocean surface. To examine the time dependent evolution of the PDF of the surface temperature in this case, we adopt a one-dimensional model of the global surface heat balance, similar in form to previous studies (Wigley and Schlesinger, 1985; Wigley and Raper, 1990; Lindzen and Giannitsis, 1998). We initially assume a global ocean under the action of step-function 
forcing. Modifications resulting from time-dependent forcing and the inclusion of land-sea surface differences are discussed in later sections. We calculate the evolution of $T(t)$ for fixed values of the input parameters of the model and then we find the shape of the PDF, $h_{T}(T, t)$, that is created by uncertainties in these input parameters and/or uncertainty in the radiative forcing. We will show that a few general ideas can guide predictions of this time dependent shape, and in particular the evolution of its high temperature tail.

\section{The Ocean Model}

In our model the ocean has a simple one-dimensional structure, shown in Figure 2: at the surface a well-mixed layer of depth $h$, density $\rho$, specific heat $C_{p}$, and thermal conductivity $\kappa[W / m-K]$ exchanges energy with the atmosphere and loses heat to the ocean below. Vertical heat transport throughout the ocean is represented by a diffusivity $\chi\left[\mathrm{m}^{2} / \mathrm{s}\right]$ and an upwelling velocity $w$, both considered constant with depth and time.

Thus, assuming a global ocean, our energy balance model consists of equations for $T(t)$, the mean time dependent mixed layer temperature anomaly (departure from its value at time $\mathrm{t}=0$ ), and $T_{t h}(z, t)$, the mean time dependent temperature anomaly at depth $z$ below the mixed layer in the deep ocean.

The mixed layer temperature obeys the equation

$$
\rho C_{p} h \frac{d T}{d t}+\frac{T\left(1-f_{a}\right)}{\lambda_{0}}-\left.\kappa \frac{\partial T_{t h}}{\partial z}\right|_{z=0}=\Delta R_{F}(t)
$$

Here $\Delta R_{F}(t)$ represents the downward radiative flux anomaly at the top of the atmosphere; $f_{a}$ is the sum of atmospheric and surface feedback factors ( for example, albedo and cloud feedbacks. 
For earlier derivations of equation 2 and discussion of the feedback factors see RB07; Roe, 2008 and references therein.). The term with $\kappa$ is the heat loss from the mixed layer to the cooler water below.

The temperature in the deep ocean obeys the equation

$$
\frac{\partial T_{t h}}{\partial t}=\chi \frac{\partial^{2} T_{t h}}{\partial z^{2}}-w \frac{\partial T_{t h}}{\partial z}
$$

Here $w<0$; it is the upwelling velocity, so that if the ocean temperature decreases with $\mathrm{z}$ (distance below the surface), the last term on the RHS of (3) is negative.

(2) and (3) nondimensionalize to

$$
X \frac{d T}{d \hat{t}}+T\left(1-f_{a}\right)-\left.f_{o} \frac{\partial T_{t h}}{\partial \hat{z}}\right|_{\hat{z}=0}=\Delta R_{F}(\hat{t})
$$

and

$$
\frac{\partial T_{t h}}{\partial \hat{t}}=\frac{\partial^{2} T_{t h}}{\partial \hat{z}^{2}}-\frac{\partial T_{t h}}{\partial \hat{z}}
$$

where $X \equiv \tau_{1} / \tau_{0}$, where $\tau_{1} \equiv \rho C_{p} h \lambda_{0} ; \tau_{0} \equiv 4 \chi / w^{2}$, and $f_{o} \equiv \rho C_{p} \lambda_{0} w ; \hat{t} \equiv t / \tau_{0} ; \hat{z} \equiv z / D ; D \equiv$ $-\chi / w$. Thus the parameters $X, f_{a}$ and $f_{o}$ determine the solution. Note that $f_{o}$ is negative. Table 1 shows that for typical values of the physical parameters, $\tau_{1} \sim 3 \mathrm{yrs}, \tau_{0} \sim 2500 \mathrm{yrs}, f_{o} \sim-0.16$, and $X \sim 0.0039$.

In this analytic model the ocean depth is infinite; as we will show, this simplification does not substantially impact the calculated surface temperature evolution or its PDF for several centuries and enables an analytic solution to (2) and (3), given in Appendix A. The boundary conditions at upper and lower boundaries are $T_{t h}(0, t)=T(t), T_{t h}(\infty, t)=0$.

The system behavior is most clearly illustrated by the response to a step function in forcing for fixed 
values of the input parameters. Figure 3 shows the solution for the three terms on the left hand side of (2) whose nondimensional equivalents are obvious in (4). The rate of change of mixed layer temperatures is initially large but then quickly declines to small values, consistent with the small value of $X$ compared to the other nondimensional numbers. As will be seen however, this small residual of gradual warming is crucial for subsequent evolution of the PDF over time. The step function in forcing is predominantly balanced by the two other factors: the heat driven down into the deep ocean, which declines slowly over time as the temperature contrast between the deep ocean and the mixed layer declines; and the outgoing longwave radiation (modified by the atmospheric feedbacks), which increases slowly over time as the surface temperature continues to increase.

\subsection{The time dependent solution: comparison of numerical and analytical re- sults}

Since we have assumed the ocean is of infinite depth, our model overestimates the strength of the oceanic heat loss at large times. To explore its range of validity we compare the analytic solution to (4) and (5) with the solution obtained from a numerical model.

Equations (2) and (3) can be readily solved numerically for fixed values of the system parameters for an ocean bottom at a depth $L$ and zero-flux boundary condition at this ocean floor. We also include heat transfer $\rho C w\left(T-T_{t h}(z=L)\right)$ directly from the surface to the deepest ocean level, which emulates bottom water formation bringing surface anomalies to depth (Hoffert et al., 1980). The numerical solution can be compared with that of the analytical model with infinite ocean depth. Since we are interested in the evolution of the PDF shape, we perform this comparison for 1000 Monte Carlo calculations, varying the value of the atmospheric feedback factor, $f_{a}$, assuming its uncertainty is governed by a Gaussian distribution with parameters given by Table 1 (as determined 
from literature surveys). We initially only consider uncertainty in atmospheric feedbacks, in order to cleanly isolate the role of the ocean heat uptake. Later in the paper we also vary ocean parameters. Our assumption that the physical parameters are normally-distributed (except where prohibited on physical grounds - for instance $f_{a}$ must be less than 1 ) has only a minor impact on the detailed shape of the resulting PDF of surface temperature, and no impact on the basic properties, trends and sensitivities of this shape, which are our focus here.

A comparison of the numerical solution with $L=4 \mathrm{~km}$ to the analytical solution with $L \rightarrow \infty$ is shown in Figure 4. The analytical and numerical solutions of the PDF are almost identical for the first five hundred years, demonstrating that the influence of ocean bottom has not yet affected the evolution of the surface temperature. It is also clear that the full range of climate sensitivity does not come close to being realized for a very long time. In this sense transient climate change is much more predictable than climate sensitivity.

There are three time scales evident in Figure 4: a fast time scale of the adjustment of the mixed layer, $\tau_{1}$; a medium time scale of the advective-diffusive adjustment $\tau_{0}$, which can be interpreted as the ratio of the thermal reservoir of the deep ocean contained in one diffusive length scale divided by the rate of upwelling of heat; and lastly a long time scale, ultimately the advective-diffusive filling of the entire deep ocean. The physical processes involved in the ultimate adjustment of the deep ocean are poorly known, and our model is not intended to represent them accurately. Nonetheless, we see that: (i) over the first few centuries, the agreement between the numerical and analytical solutions means we can proceed using the analytical model to explore the dependency of the solution on model parameters, making use of the speed of computations this model provides; (ii) the equilibrium response, and hence the equilibrium distribution of climate sensitivity, is not an issue of concern for policymakers. 


\section{Analysis: ocean heat uptake as a transient negative feedback}

Although the equilibrium (i.e., infinite time) solution for our simple analytical model is nonphysical, it is instructive to examine its behavior as we begin our investigation of $h_{T}(T, t)$. From Appendix A we find for fixed $f_{a}, f_{o}$ that

$$
\begin{array}{r}
T(\infty) \equiv T_{\infty}=\frac{T_{0}}{\left(1-f_{a}-f_{o}\right)} \\
T_{t h}(z, \infty)=T_{\infty} .
\end{array}
$$

For this equilibrium solution, then, the oceanic heat uptake indeed behaves like a negative feedback of strength $f_{o}$. For the standard parameters in Table $1, f_{o} \approx-0.15$. Therefore the equilibrium PDF for the model would have the shape given by equation (1), with $\bar{f}=\bar{f}_{a}+\bar{f}_{o} \approx 0.5$ and $\sigma_{f}$ the variance in the total feedback. The resulting distributions are shown in Figure 1 in light colors: the addition of a negative feedback moves the PDF further from the limit of $f \rightarrow 1$, reducing the skewing tendency on the temperature response. Probabilities are therefore concentrated more nearly about the mean (and weaker) temperature response. This result can be understood physically: the effect of the radiative forcing on the mixed layer is damped by the heat that is taken up by the deep ocean. The greater the surface temperature change, the more heat is driven into the deep ocean, and the greater degree to which the effect of the radiative forcing is damped. Thus this effect of the ocean heat uptake is similar to that of a negative feedback.

We now show that, on the century timescale, the impact of heat storage in the ocean on the surface temperature (and hence on its PDF) is also very similar to that of a transient negative feedback, delaying the warming of the mixed layer. As in Figure 4, we run Monte Carlo simulations of the analytic solutions to our model equations (4) and (5), again varying the value of the atmospheric

feedback factor, $f_{a}$, and using the standard values of $f_{o}$ and $X$ in Table 1 . Figure 5a shows the 
evolution of $h_{T}(T, t)$ over the first five hundred years after the step-function increase in forcing, calculated from 10,000 Monte Carlo calculations. Within approximately 200 years the PDF asymptotes to the equilibrium PDF expected given the uncertainty in the atmospheric feedbacks, modified by the inclusion of the negative feedback due to the ocean heat uptake (i.e., (1) with $f=f_{a}+f_{o}$ ). The same is of course true for the cumulative distribution function $p_{\text {cum }}\left(T_{c}, t\right) \equiv \int_{T c}^{\infty} h_{T}(T, t) d T$, shown in Figure 5b.

\subsection{Quantifying the transient ocean feedback}

Figure 5a shows that even during the first hundred years of the transient climate evolution the PDF $h_{T}(T, t)$ is skewed, and similar in functional form to the equilibrium climate sensitivity PDF (i.e., (1) with total feedback $f=f_{a}+f_{o}$ ). Moreover, as shown in Figure 3, the rate of heat uptake of the ocean is large initially, but diminishes rapidly with time. These two observations suggest that the ocean heat uptake can be characterized as a negative feedback that is initially very strong, that decreases over the medium term (i.e., several hundred years) to that given by $f_{o}$, and ultimately diminishes to zero as true equilibrium is reached.

To quantify this behavior, at each time-step in the integration we calculate the probability density and temperature at the mode of the evolving PDFs in Figure 5a, and use (1) to determine the value of $\bar{f}$ and $\sigma_{f}$ of the equilibrium PDF that would match that mode. We then calculated the

r.m.s. error between the actual PDF at that time with the equilibrium PDF that the matched $\bar{f}$ and $\sigma_{f}$ would generate. These calculations are shown in Figure 6. To a high degree of accuracy the evolving PDF can be well characterized by (1) with an effective negative ocean feedback that is strong initially, and diminishes over the medium term to its analytical value. The implied spread of uncertainty in the feedbacks (i.e., $\sigma_{f}$ ) stays approximately constant with time (Figure 6c). 


\subsection{The conservation equation for $h_{T}(T, t)$ and the growth of the fat tail}

Our goal in this paper is to describe the shape of $h_{T}(T, t)$ as it evolves over the next centuries, as shown in Figures 4 to 6 . Physical understanding of this evolution is aided by noting that $h_{t}(T, t)$ obeys a conservation equation analogous to that of the density in a compressible fluid in which there are no diffusive processes:

$$
\frac{\partial h_{T}(T, t)}{\partial t}=-\frac{\partial\left(h_{T}(T, t) \dot{T}\right)}{\partial T}
$$

where $\dot{T}$ is the average rate of warming of the climate states close to a given $T$. As such, $\dot{T}$ can be thought of as the mass-weighted warming 'velocity' of a given point on the PDF. Therefore $h_{T} \times \dot{T}$ is a density of probabilities multiplied by a velocity of probabilities, and so is equal to the flux of probabilities from lower to higher temperatures. Figure 7 shows the terms going into this probability flux. The right hand side of (7) is the convergence of that flux. The shape of the PDF changes with time, as the probability density increases where this convergence is positive and decreases where it is negative.

The rate of increase with time of the cumulative probability that $T$ exceeds any given $T_{c}$ is a measure of the growth of the tail, and can be found by integrating (7) from a particular value of $T, T=T_{c}$, to $\infty$. Taking account the fact that $h_{T}(T, t) \rightarrow 0$ as $T \rightarrow \infty$ gives

$$
\frac{\partial p_{\text {cum }}\left(T_{c}, t\right)}{\partial t}=\left.h_{T}\left(T_{c}, t\right) \dot{T}\right|_{T=T_{c}}
$$

A measure of the rate of spreading of the high temperature tail of the PDF is $U_{\text {spread }}(t) \equiv$ $\dot{T}(t)_{\text {pcum }=.5}-\dot{T}(t)_{\text {pcum=.05 }}$. As shown in Figures 4,5 , and $7 \mathrm{~b}, U_{\text {spread }}(t)$ is large in the first decades and decreases thereafter as the PDF approaches the equilibrium shape (i.e., that given by (1)) corresponding to total feedback $f=f_{a}+f_{o}$. 


\section{Some policy-related issues}

Our simple framework can provide insight into several issues of current interest to climate policy makers. The shape and evolution of the PDF of the climate response is a critical factor in economic planning and of great current interest (e.g., Weitzman, 2008a,b). We focus here on the following:

1. How well can we predict when the temperature will reach a given value?

2. Which parameters dominate the uncertainty? Does it help more to reduce uncertainties in the atmospheric feedbacks or to reduce those related to the ocean feedback?

3. How does uncertainty in climate forcing affect predictions of climate change?

4. How does the choice of emissions scenario affect the probability of reaching 'dangerous' climate change? And how does uncertainty in anticipated emissions compare with uncertainty in climate parameters? In other words, what is our best strategy to improve predictability of avoiding a 'dangerous' climate change?

In order to study these practical problems in climate prediction we must make a realistic approximation to expected forcing. The step function approximation for the radiative forcing has provided a simple tool with which to investigate the ways that uncertainties in physical factors impact the time dependence of the PDF of the surface temperature. However, in reality the forcing is expected to rise steadily with time for the next decades, and we now examine the evolution of the temperature PDF for this case.

The simplest approximation is that the forcing grows linearly with time. We first predict some features of the PDF of surface temperature that will result from a known $\Delta R_{F}(t)=V_{F} t$; in a later 
section we consider the impacts of uncertainty in the parameter $V_{F}$. Figure 8a,b show the PDF and CDF of the climate response to a ramp forcing, given uncertainty in $f_{a}$.

Equations (7) and (8) characterize the evolution of the PDF and CDF of temperature under ramp forcing. The terms in the probability flux are shown in Figure 9 , and $U_{\text {spread }}(t)$ can be diagnosed from Figure $9 \mathrm{~b}$. In contrast to the step function case, this spreading rate now increases with time, due to the acceleration of the high temperature tail. As before, because of the smallness of the parameter $X$, the explicit time dependent term in (2) is small compared with the other terms so that for any $V_{F}$ the sum of the second and third terms in this equation should be approximately linear in time.

As a rough approximation to the temperature evolution on a trajectory with fixed $\left(f_{a}, f_{o}\right)$ after the first fifty years or so is, $T(t) \approx \lambda_{0} V_{F} t /\left(1-f_{a}-f_{o}\right) \equiv \lambda_{0} V_{F} t /(1-f)$. The relative uncertainty in temperature associated with relative uncertainty in feedback $\delta f / \bar{f}$ is $\delta T(t) / \bar{T}(t) \approx \delta f(1-\bar{f})^{-1}$, independent of $V_{F}$. For our standard parameters, this relative error is approximately 0.3.

\subsection{How well can we predict when the temperature will reach a given value?}

From (7) it follows that the PDF of the time $t_{c}$ it takes to reach a given value $T=T_{c}$ is

$$
h_{t}\left(t_{c}, T_{c}\right)\left[s^{-1}\right]=\frac{1}{p_{\text {cum }}\left(T_{c}, \infty\right)}\left(\left.\dot{T}\right|_{T=T_{c}} h_{T}\left(T_{c}, t_{c}\right)\right) .
$$

This PDF is shown for ramp forcing in Figure (10). It broadens as the threshold temperature increases. Thus predicating a policy choice on a threshold of $T_{c} \geq 2{ }^{\circ} \mathrm{C}$ must take into account the considerable uncertainty in the time that this temperature (or any given temperature) will be reached, even if there is no uncertainty in the forcing. 


\subsection{Which model parameters dominate the uncertainty?}

RB07 showed that the fat tail of the equilibrium PDF of climate sensitivity is relatively insensitive to a reduction of uncertainty in the feedbacks. The analytical model developed in this study allows very efficient Monte Carlo estimates of this reduction for the evolving PDF. We first perform simulations in which model parameters $f_{a}, h, \chi$, and $w$ are assumed to vary randomly within normal distributions governed by Table 1. No great effort was made to optimize them to the best possible values - the purpose of these calculations is illustrative.

Figure 11 shows the evolving PDF of the response to a linear ramp forcing of $4 \mathrm{~W} \mathrm{~m}^{-2} 100 \mathrm{yr}^{-1}$, estimated from 10,000 Monte Carlo calculations, and for various combinations of parameter uncertainty. With all uncertainties included the $95 \%$ confidence interval at 200 years is approximately $2{ }^{\circ} \mathrm{C}$ to $5{ }^{\circ} \mathrm{C}$. Since the total uncertainty is the sum of the uncertainties in the atmospheric and the oceanic parameters, reducing the uncertainty in $f_{o}$ by a given amount has the same impact on the $\mathrm{PDF}$ as does the same reduction of uncertainty in $f_{a}$ by the same amount even though $\bar{f}_{o}<\bar{f}_{a}$. However, because of the differences in magnitudes of the two means, reducing $\sigma_{o}$ and $\sigma_{a}$ by the same amount requires a much greater reduction in $\sigma_{o} / \bar{f}_{o}$ than in $\sigma_{a} / \bar{f}_{a}$.

The presence of the negative ocean feedback in the transient evolution of the system means that the total feedback (i.e., $f_{a}+f_{o}$ ) is shifted away from the $f=1$ limit. Consequently the effect of the $1 /(1-f)$ stretching that creates the fat tail for large climate responses is significantly reduced, and probabilities are much more concentrated around the mean value (Figure 1). Hence, in contrast to the equilibrium climate sensitivity results of RB07, a reduction of uncertainty in the atmospheric feedbacks does have a significant effect on reducing uncertainty in the evolving climate response. It is also worth noting that if uncertainty in different model parameters are not formally independent 
of each other: if uncertainty in one parameter is reduced, it is possible that leads to a reduction uncertainty in other parameters when the model is evaluated against observations (Knutti, 2002, 2003; Forest et al., 2002).

\subsection{How does uncertainty in climate forcing affect projections of climate change?}

Heretofore we have assumed the forcing is perfectly known. However there are substantial uncertainties in forcing at the present day, and also uncertainties in what future emissions and the consequent climate forcing will be (e.g., IPCC07). Although it is beyond the scope of this paper to review these uncertainties and their likely evolution, we can roughly estimate their impacts on the evolving PDF of surface temperature. Here we assume the mean forcing is a known linear function of time, $\Delta R_{F}(t)$, with symmetrically-distributed uncertainty $\sigma_{R}$ about this mean, where $\sigma_{R} \ll \Delta R_{F}(t)$. In a later section we explore the impacts on climate predictability of the uncertainty associated with choice of mean forcing $\Delta R_{F}(t) . \sigma_{R}$ is independent of time, of temperature, and of the various feedbacks in this exercise.

\subsubsection{Impact on the equilibrium climate change}

It is instructive to examine the relative impacts of uncertainties in climate forcing and in climate feedbacks on the equilibrium response to step function forcing, and then to apply those results to the case of time dependent forcing. For the former we have $h_{T}(T) d T=h_{f}\left(f_{a}\right) d f_{a} \times h_{R}\left(\Delta R_{F}\right) d\left(\Delta R_{F}\right)$, where $h_{f}$ and $h_{R}$ are the PDFs of $f_{a}$ and $\Delta R_{F}$, respectively. Using the fact that $T=\lambda_{0} \Delta R_{F} /(1-$ $f_{a}$ ), and integrating over $\Delta R_{f}$ from zero to infinity gives

$$
h_{T}(T)=\lambda_{0} \int_{0}^{\infty} h_{f}\left(1-\frac{\lambda_{0} \Delta R_{F}}{T}\right) \cdot h_{R}\left(\Delta R_{F}\right) \cdot \frac{\Delta R}{T^{2}} \cdot d\left(\Delta R_{F}\right) .
$$


For a normal distribution of uncertainty in forcing, with mean value $\Delta \bar{R}_{F}=4 \mathrm{~W} \mathrm{~m}^{-2}$ and $\sigma_{R}=$ $1 \mathrm{~W} \mathrm{~m}^{-2}$, the PDF of the climate response is shown in Figure 12. The uncertainty in forcing does very little to the likelihood of large temperature increases since the equilibrium PDF varies little with temperature in that regime. It does, however, redistribute likelihood away from the center of the PDF towards lower climate responses. Thus uncertainty in forcing is more important than uncertainty in feedbacks at lower temperatures and less important at higher temperatures. For the values chosen in this study, the cross over in relative importance is near $T \sim 2{ }^{\circ} \mathrm{C}$, which can be established from (10).

\subsubsection{Impact on the transient climate change}

Now we assume ramp forcing with mean value $\Delta \bar{R}_{F}(t)=\bar{V}_{F} t, V_{F}=4 \mathrm{~W} \mathrm{~m}^{-2} 100 \mathrm{yr}^{-1}$ and add in an uncertainty in $V_{F}$ of $\sigma_{V_{F}}=0.25 \bar{V}_{F}$, which approximately agrees with present estimates of the forcing uncertainty (IPCC07). A comparison of the light and dark shading in Figure 8a shows the impact of forcing uncertainty on the evolving response. Contrary to the case of the equilibrium PDF to a step forcing shown above, there is now some significant effect at high climate responses. For time dependent forcing, the small value of the nondimensional parameter $X$ in (4) leads to an approximately linear relationship between forcing and response; lower forcing produces

more gradual warming. As in the case of the equilibrium response to step forcing, the impact of symmetric forcing uncertainty is to add slightly greater probability of low temperature response than high climate response, leading to a less skewed PDF with the forcing uncertainty than without. 


\subsection{How does the choice of emissions scenario affect the probability of reaching 'dangerous' climate change?}

In this section we consider those factors determining the probability that the maximum global mean temperature will reach (or remain less than) a pre-set threshold, if we manage to eventually reduce forcing toward pre-industrial levels. We assume the forcing is a quadratic function of time, characterized by two parameters: a maximum forcing and the time taken to reach that maximum. As a proxy for climate forcing, we assume that the concentration of $\mathrm{CO}_{2}$ is related to the radiative forcing $\Delta R_{f}$ via the relationship $\Delta R=5.35 \times \log _{\mathrm{e}}\left(\mathrm{CO}_{2} / 280\right) \mathrm{W} \mathrm{m}{ }^{-2}$, where $\mathrm{CO}_{2}$ is measured in ppmv (Ramaswamy et al., 2001). Thus, whereas stabilization of forcing at some level leads to the eventual full PDF characterized by (1), including the full width of the fat tail, we now consider scenarios in which forcing $\left(\mathrm{CO}_{2}\right.$ concentrations) comes back down towards pre-industrial. In these cases the fat tail of the equilibrium PDF does not have time to develop in the climate projections, and this causes a striking reduction in the risk of experiencing the extreme climate responses, a risk that cannot be ruled out as an ultimate equilibrium response to a stabilization scenario.

Figure 13 shows some examples of the forcing scenarios and the climate response to them, assuming the standard set of parameters. The maximum temperature is reached between 20 and 40 years after the maximum in concentrations, depending on the rate of decline in the concentrations. Note that this figure is consistent with the arguments of Cox and Stephenson (2007) who point out that uncertainty in emissions becomes more important than uncertainty in processes and parameters.

We consider a wide range of $\mathrm{CO}_{2}$-equivalent concentration scenarios spanning maximum-concentrations of between 280 ppmv (i.e., pre-industrial levels) and 1100 ppmv, and spanning time-to-maximum of between ten and five hundred years. 
We emphasize that we do not consider the modeled climate responses to be optimized climate projections for the given scenarios. The purpose of the calculations is two-fold: firstly to show the ability of the model framework to make reasonable probabilistic forecasts for different concentration scenarios; and secondly, to explore how reducing uncertainty in model parameters changes the projected PDF of the climate response to different emissions scenarios. We note also that for these simulations we have assumed an aquaplanet, so the temperatures refer to the ocean mixed layer temperature on such a planet. We discuss the impact of including a land fraction below.

Figure $14 \mathrm{a}$ and $\mathrm{b}$ show the $5 \%$ and $95 \%$ bounds on the PDF of the maximum temperature reached for different concentration scenarios, all of the form shown in Figure ?? The asymmetry of the PDFs is reflected in Figure 14, in that the upper 95\% bound is more sensitive to variations in the maximum concentration than is the lower bound. Figure 14c and d show the effect of halving uncertainty in all model parameters on the $5 \%$ and $95 \%$ bounds of the climate projections. The range of climate projections for any given concentration scenarios narrows in response to the reduced uncertainty. However the $95 \%$ bound on the maximum temperature reached (i.e., panels a and c) is most sensitive to small changes in the maximum concentration reached.

As a guide for future climate projections, in Figure 14 we have superimposed (green arrows) the low, mid, and high values of $\mathrm{CO}_{2}$-equivalent concentrations projected for the year 2100 in IPCC07. Assuming the concentrations decline after 2100, the Figure allows us to estimate the bounds on the PDF of maximum temperature each of these IPCC scenarios would produce. The uncertainty in concentration scenarios encompass approximately a factor of two uncertainty in the climate response and therefore, at this point in time, dominate over parameter uncertainty in generating uncertainties in climate projections. 


\section{$5 \quad$ Limitations of Our Analysis}

\subsection{The Impact of Land and Regional Variations}

As has been shown by several authors (e.g., North and Coakley, 1979; Wigley and Schlesinger, 1985; Lindzen and Giannitsis, 1998), the impact of the land fraction of the global surface on the evolving global surface temperature can be approximated by replacing $X$ by $\gamma X$, and $f_{o}$ by $\gamma f_{o}$ in equation (4) for the mixed layer temperature, where $\gamma$ varies between about 0.7 to 1.0, depending on the strength of the land-ocean coupling assumed. The global mean surface temperature is then an area weighted sum of the land temperature and this mixed layer temperature. We can expect that the change $X \rightarrow \gamma X$ will not have much impact but the diminished magnitude of the effective oceanic feedback $f_{o} \rightarrow \gamma f_{o}$ will lessen the role of the ocean in holding back the surface warming. The PDF of the evolving global surface temperature is then peaked at slightly higher temperatures than for the aquaplanet at all times, and the inclusion of the land factor somewhat accelerates growth of the tail of the PDF (not shown).

It is important to appreciate that there are possibly large local departures from the global picture presented here. For instance, although the global feedback factor for surface albedo is small compared to, for example, that for water vapor (e.g., Hansen et al., 1984), the presence or absence of sea-ice may be the dominant control on climate in the vicinity of the sea ice margin. Another case in point is that, in contrast to our globally-averaged model framework, ocean heat uptake is a strongly regional phenomenon, and characteristic climate variability can be quite strongly affected in regions where the presence of a deep, well-mixed column of ocean water increases the effective thermal inertia (e.g., Manabe and Stouffer, 1996). Nonetheless the ability of such simple models to emulate global mean temperatures both in observations and in output from coupled global climate 
models suggests that such regional-scale effects do not overwhelm the global-scale picture.

\subsection{Nonlinear feedbacks and missing feedbacks}

For any given climate trajectory we assumed that the feedbacks were constant. In fact some modeling studies suggest some dependence of feedbacks on mean climate state (i. e., global average surface temperature) (e.g., Colman et al., 1997), though the results vary widely: Watterson (2000) finds very little change in climate sensitivity with climate state; Senior and Mitchell (2000) find the effective climate sensitivity increasing by $40 \%$ after 800 years of a climate change scenario; Boer and $\mathrm{Yu}$ (2003) find a 10-20\% decrease in a similar time frame. Note that these last two results are consistent with an expectation from (1) that one would expect larger changes for an increase in effective climate sensitivity than for a decrease in climate sensitivity. We have crudely emulated these results by imposing climate-dependent feedback strength (i.e., $f_{a}=f_{a}(T)$ ), but over the first few centuries, these changes cause only small quantitative differences in the evolving PDF (not shown).

It should also be noted that we have also left out some other feedbacks, such as changes in the strength of the thermohaline circulation (e.g., Wood et al., 2003) or the carbon cycle (e.g., Torn and Harte, 2006), which have been suggested to be important. Simple characterizations of such feedbacks could readily be incorporated in the framework presented here. 


\section{Conclusions}

We have investigated controls on the shape of the PDF of the global mean temperature response to climate forcing, as it evolves in time. In particular we have examined how uncertainties in physical feedbacks and uncertainties in climate forcing combine to affect uncertainty in the evolving climate response.

A principal result is that the ocean heat uptake can be well characterized as a transient negative feedback that operates out to at least few centuries. The effect of this negative feedback is that the PDF of the transient climate change is much narrower and less skewed than the PDF of the equilibrium climate response. In this sense, transient climate change is much more predictable than equilibrium climate sensitivity. Another result that carries some important policy implications is that the larger the temperature change that is being contemplated, the more uncertain is the timing of when that temperature will be reached.

We also showed that as the transient ocean feedback declines, the fat tail of probabilities of large climate change grows exceedingly gradually, as the system slowly trends towards equilibrium. The protracted development of the fat tail can be represented as a flux of probabilities that depends on the warming rate and the probability density, both of which have very low values in the tail. The fact it takes so long for the full range of possible climate states to be realized suggests that further efforts to constrain the fat tail of equilibrium climate sensitivity will be of very limited value for climate projections on societally-relevant timescales.

For both the transient and equilibrium climate response, introducing uncertainty in the forcing has the effect of broadening the PDF of the climate response, and also of making the PDF more 
symmetric, which increases the probability of lower climate changes.

The combined atmosphere feedbacks $\left(f_{a}\right)$ are larger in magnitude than the ocean heat uptake feedback $\left(f_{o}\right)$. Thus, to the extent that progress is possible towards reducing uncertainty in climate response, reducing uncertainty in atmospheric feedbacks by a given percentage has a bigger impact than reducting uncertainty in ocean feedbacks by the same percentage. However, and importantly for future climate projections, the uncertainties in climate feedbacks are dwarfed by the uncertainty in future climate forcing, primarily due to uncertainty in anthropogenic emissions (Figure 14).

We've used a very basic model of the climate system. However we believe that the basic physical tendencies evinced here will also apply in more sophisticated and complete physical models of climate prediction. RB07 showed that (1) characterizes the behavior of full-physics global climate models, and that it spans the full range of climate sensitivities arising out of multi-thousand member ensembles of climate model integrations. There is also no doubt that ocean heat uptake acts to buffer the response of the surface temperature to climate forcing, and the values of model parameters that we use are consistent with previous studies that have been calibrated to match observations. While considerable uncertainty surrounds the processes governing the long term adjustment of the ocean, our results out to a few centuries are quite insensitive to variations in the ocean heat uptake (e.g., Figure 11).

Our results suggest that once feedback factors have been determined, reasonable estimates of the evolving PDF of future climate (at least as characterized by global mean temperature) can be obtained without recourse to large ensemble integrations of global climate models. Such simple calculations are powerful and efficient tools for exploring the climate response to different scenarios of future anthropogenic climate forcing. 


\section{Acknowledgments}

The authors are grateful for enlightening conversations with David Battisti, Reto Knutti, Eric Steig,

Rob Wood, Chris Brierly, Martin Weitzman, and Richard Lindzen. 


\section{Appendix A: The solution of Equations (4) and (5)}

We assume that the nondimensional time dependent forcing can be expressed as a polynomial

$$
\Delta R_{F}(\hat{t})=\sum_{i=0} c_{i} \hat{t}^{i}
$$

and that the forcings are sufficiently small that the resulting temperatures are sums from the individual contributions:

$$
\begin{aligned}
T(\hat{t}) & =\sum_{i} T_{m l}^{i}(\hat{t}), \\
T_{t h}(\hat{z}, \hat{t}) & =\sum_{i} T_{t h}^{i}(\hat{z}, \hat{t}) .
\end{aligned}
$$

Thus the solutions for all forcings are defined by the nondimensional parameters $X, f_{a}$ and $f_{o}$ and the coefficients $c_{i}$.

\section{A-2 Solution}

Because of the linear nature of this model, the temperature in the deep ocean is related to that of the mixed layer by

$$
T_{t h}(\hat{z}, \hat{t})=\int T\left(\hat{t}_{0}\right) \frac{\hat{z}}{\left(\hat{t}-\hat{t}_{0}\right)^{1.5}} \operatorname{Exp}\left[-\frac{\left(\hat{z}+4\left(\hat{t}-\hat{t}_{0}\right)^{2}\right)}{16\left(\hat{t}-\hat{t}_{0}\right)}\right] d \hat{t}_{0} .
$$

\section{A-3 Step function forcing}

In the special case of step function forcing, (assuming forcing due to doubling of $\mathrm{CO}_{2}$ ), $c_{0}=$ $\lambda_{0} \Delta R_{F}\left(2 \times \mathrm{CO}_{2}\right), c_{i}=0, i \neq 0$. 
Using LaPlace transform methods, it can easily be shown that the solution is of the form

$$
T_{m l}^{0}(\hat{t})=c_{0}(G[\eta=0, \hat{t}]+I[\hat{r}=-1, \hat{t}])
$$

where

$$
G[\eta, \hat{t}] \equiv \frac{\exp [\eta \hat{t}]}{\left(\eta X+s+0.5 f_{o}(1+\eta)^{0.5}\right)}
$$

$s \equiv 1-f_{a}+0.5 f_{o}$, and

$$
\begin{aligned}
I[\hat{r}, \hat{t}] & \equiv-\int_{0}^{\infty} \frac{\alpha^{0.5} \exp [-\alpha \hat{t}]}{(\hat{r}+\alpha)\left(\alpha-\hat{r}_{1}\right)\left(\alpha-\hat{r}_{2}\right)} d \alpha \\
& =\pi\left[\frac{1}{\left(\hat{r}_{1}-\hat{r}\right)\left(\hat{r}_{2}-\hat{r}\right)} f[\hat{r}, \hat{t}]\right. \\
& +\frac{1}{\left(\hat{r}_{1}-\hat{r}\right)\left(\hat{r}_{1}-\hat{r}_{2}\right)} f\left[\hat{r}_{1}, \hat{t}\right] \\
& \left.+\frac{1}{\left(\hat{r}_{2}-\hat{r}\right)\left(\hat{r}_{2}-\hat{r}_{1}\right)} f\left[\hat{r}_{2}, \hat{t}\right]\right]
\end{aligned}
$$

where $f[x, \hat{t}] \equiv \frac{f_{o}}{2 \pi X^{2}} \exp [-\hat{t}](-x)^{0.5} \exp [-x \hat{t}] \operatorname{Er} f c\left[(-x \hat{t})^{0.5}\right]$. The nondimensional roots $\hat{r}_{1}, \hat{r}_{2}$ are

$$
\hat{r_{1}}, \hat{r_{2}}=\frac{1}{2 X}\left(-\left(-2 s+\frac{f_{o}^{2}}{4 X}+2 X\right) \pm \sqrt{\left(-2 s+\frac{f_{o}^{2}}{4 X}+2 X\right)^{2}-4(X-s)^{2}}\right) .
$$

\section{A-4 Time dependent forcing}

The contribution of the forcing term $c_{i} \hat{t}^{i}$ to the mixed layer temperature is

$$
T_{m l}^{i}(\hat{t})=c_{i}\left(\left.\frac{\partial^{i} G[\eta, \hat{t}]}{\partial \eta^{i}}\right|_{\eta=0}+\left.\frac{\partial^{i} I[\hat{r}, \hat{t}]}{\partial \hat{r}^{i}}\right|_{\hat{r}=-1}\right)
$$




\section{References}

Allen, M.R., and D.J. Frame, 2007: Call off the quest. Science, 318, 582-583.

Arhennius, S., 1896: On the influence of carbon acid in the air upon the temperature of the ground. Philosoph. Mag., 41, 237-276.

Boer, G. J., and B. Yu, 2003: Climate sensitivity and climate state. Climate Dyn., 21, 167176.

Cess, R. D., 1975: Global climate change: An investigation of atmospheric feedback mechanisms. Tellus, 27 , 193-198.

Colman, R., S. Power, and B. McAvaney, 1997: Non-linear climate feedback analysis in an atmospheric GCM. Climate Dyn., 13, 717-731.

Colman, R., 2003: A comparison of climate feedbacks in general circulation models. Climate Dyn., 20, 865-873.

Cox, P., D. Stephenson, 2007: A changing climate for prediction. Science, 317, 207-208.

Forest, C.E., P.H. Stone, A.P. Sokolov, M.R. Allen, and M.D. Webster, 2002: Quantifying uncertainties in climate system properties with the use of recent climate observations. Science, 295, 113117.

Forest, C. E., P. H. Stone, and A. P. Sokolov, 2006: Estimated PDFs of climate system properties including natural and anthropogenic forcings. Geophys. Res. Lett., 33, L01705, doi:10.1029/2005GL023977.

Frame, D.J., B.B.B. Booth, J.A. Kettleborough, D.A. Stainforth, J.M. Gregory, M. Collins, and M.R. Allen, 2005: Constraining climate forecasts: The role of prior assumptions, Geophys. Res. Lett., 32, L09702, doi:10.1029/2004GL022241. 
Frame, D.J., D.A. Stone, P.A. Stott, and M.R. Allen, 2006: Alternatives to stabilization scenarios, Geophys. Res. Lett., 33, L14707, doi:10.1029/2006GL025801.

Hansen, J., A. Lacis, D. Rind, G. Russell, P. Stone, I. Fung, R. Ruedy, and J. Lerner, 1984: Climate sensitivity: Analysis of feedback mechanisms. In Climate Processes and Climate Sensitivity, Geophys. Monogr., Vol. 29, Amer. Geophys. Union, 130163.

Hansen, J., G. Russell, A. Lacis, I. Fung, D. Rind, and P. Stone, 1985: Climate Response Times: Dependence on climate sensitivity and ocean mixing. Science, 229, 857859.

Hoffert, M. I., A. J. Callegari, and C.-T. Hsieh, 1980: The role of deep sea heat storage in the secular response to climatic forcing, J. Geophys. Res., 85, 6667-6679.

IPCC, 2007: Physical Science Basis. Contribution of Working Group I to the Fourth Assessment Report of the Intergovernmental Panel on Climate Change (Cambridge Univ. Press, 2007).

Knutti, R., F. Joos, S. A. Müller, G.-K. Plattner, and T.F. Stocker, 2005: Probabilistic climate change projections for CO2 stabilization profiles, Geophys. Res. Lett., 32, L20707, doi:10.1029/2005GL023294.

Lindzen, R. S., and C. Giannitsis, 1998: On the climatic implications of volcanic cooling, J. Geophys. Res., 103, 59295941.

Manabe, S, and R.J. Stouffer, 1996: Low frequency variability of surface air temperature in a 1000-year integration of a coupled atmosphere-ocean-land surface model. J. Climate, 9, 376393.

Maxwell, J.C., 1867: On Governors. Proc. Royal Soc., 16, 270-283.

North, G. R., and J. A. Coakley Jr., 1979: Differences between seasonal and mean annual energy balance model calculations of climate and climate sensitivity. J. Atmos. Sci., 36, 11891204. 
Ramaswamy, V., and Coauthors, 2001: Radiative forcing of climate change. In Climate Change 2001: The Scientific Basis, J. T. Houghton et al., Eds., Cambridge University Press, 349416.

Raper, S.C.B., J.M. Gregory, and T.J. Osborn, 2001: Use of an upwelling-diffusion energy balance climate model to simulate and diagnose A/OGCM results. Clim. Dyn., 17, 601613.

Roe, G.H. and M. B. Baker, 2007: Why is climate sensitivity so unpredictable? Science, 318, $629-632$.

Roe, G.H., 2008: Feedbacks, timescales, and seeing red. Ann. Rev. Earth Plan. Sci. In review.

Schlesinger, M.E., 1985: Feedback analysis of results from energy balance and radiative-convective models. In The Potential Climatic Effects of Increasing Carbon Dioxide, M. C. MacCracken and F. M. Luther, Eds., U.S. Department of Energy, DOE/ER-0237, 280-319.

Senior, C.A, and J.F.B. Mitchell, 2000: The time-dependence of climate sensitivity. Geophys. Res. Lett., 27, 2685-2688.

Soden, B.J., and I.M. Held, 2006: An assessment of climate feedbacks in coupled oceanatmosphere models. J. Climate, 19, 3354-3360.

Torn, M.S., and J. Harte, 2006: Missing feedbacks, asymmetric uncertainties, and the underestimation of future warming, Geophys. Res. Lett., 33, L10703, doi:10.1029/2005GL025540.

Watterson, I.G., 2000: Interpretation of simulated global warming using a simple model, J. Climate, 13, 202-215.

Weitzman, M.L., 2008a: On modeling and interpreting the economics of catastrophic climate change. In preparation. 
Weitzman, M.L., 2008b: Dynamic economic consequences of the climate-sensitivity inference dilema. In preparation.

Wigley, T.M.L., and M.E. Schlesinger, 1985: Analytical solution for the effect of increasing $\mathrm{CO}_{2}$ on global mean temperature. Nature, 315, 649652.

Wigley, T. M. L., and S. C. B. Raper, 2001: Interpretation of high projections for global-mean warming, Science, 293, 451454.

Wood, R.A., M. Vellinga, and R. Thorpe, 2003: Global Warming and Thermohaline Circulation Stability Philosophical Transactions: Mathematical, Physical and Engineering Sciences. No. 1810, Abrupt Climate Change: Evidence, Mechanisms and Implications, 361, 961-1975. 


\begin{tabular}{|c|c||c|c|}
\hline Parameter & Symbol & mean value & $\pm 1 \sigma$ \\
\hline \hline Atmospheric feedback factor & $f_{a}$ & 0.65 & 0.13 \\
Mixed layer depth & $\mathrm{h}$ & $75 \mathrm{~m}$ & $25 \mathrm{~m}$ \\
Upwelling rate & $\mathrm{w}$ & $-1.3 \times 10^{-7} \mathrm{~ms}^{-1}$ & $0.5 \times 10^{-7} \mathrm{~ms}^{-1}$ \\
Ocean diffusivity & $\chi$ & $1.5 \times 10^{-4} \mathrm{~m}^{2} \mathrm{~s}^{-1}$ & $0.5 \times 10^{-4} \mathrm{~m}^{2} \mathrm{~s}^{-1}$ \\
\hline Mixed layer adjustment time & $\tau_{1}$ & 3.0 years & 1.0 years \\
Diffusive adjustment time & $\tau_{o}$ & 2500 years & 1200 years \\
Ocean feedback factor & $f_{o}$ & -0.16 & 0.06 \\
Ocean warming parameter & $X$ & 0.0039 & 0.0026 \\
\hline
\end{tabular}

Table 1: Parameter values and ranges used in the numerical and analytical models and Monte Carlo simulations. The top four are physical parameters and the lower four are derived variables for the analytical model. For Monte Carlo calculations, parameter values are drawn from normal distributions, with the given standard deviations, except for implementing the following physical constraints: $h, \chi$, and $-w$ must all be positive; and $f_{a}$ must be less than 1 . Note that here we have put $\kappa=\rho C \chi$. Parameter uncertainties are based on those reported in previous studies (Hoffert, 1980; Wigley and Schlesinger, 1985; Lindzen and Giannitsis, 1998; Raper et al., 2001; Colman, 2003; Soden and Held, 2006; Forest et al., 2002, 2006). Because ratios are taken and because of physical constraints, none of the parameters in the lower half of the table are normally distributed - so the $\pm 1 \sigma$ values given are one half of the parameter range in which $68 \%$ of the values lie. 


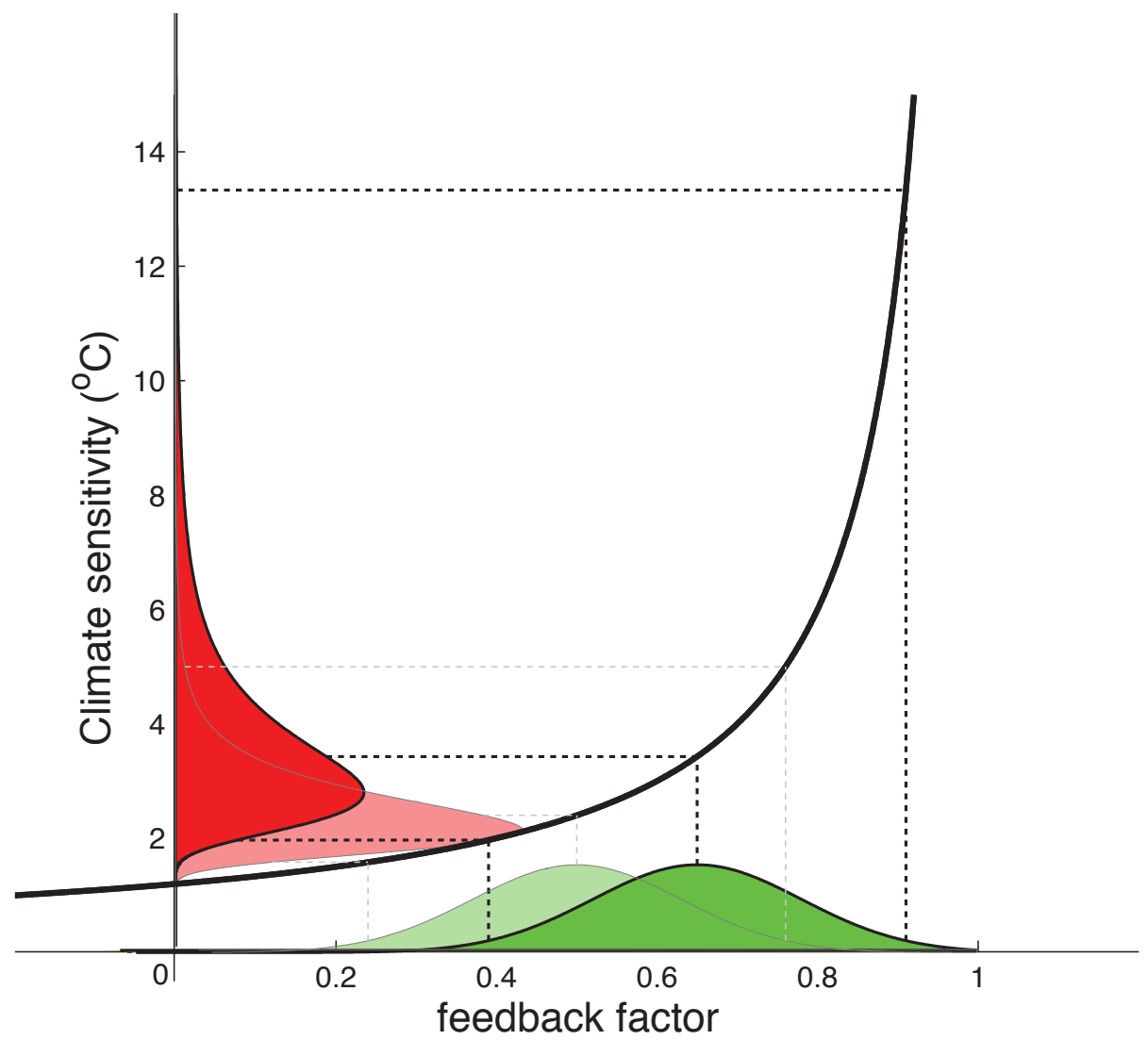

Figure 1: Illustration of the relationships between feedback factor $f$ and climate sensitivity $T$ for step function forcing. The assumed magnitude and spread in the feedback factors in the dark shading are typical for GCM integrations (e.g., Colman, 2003; Soden and Held, 2006), and lead to a strongly-skewed distribution of climate sensitivity. The dark dashed lines show the mean and 95\% bounds. The lighter shading and lines illustrates how the PDF of climate sensitivity changes when the mean feedback is displaced by -0.15 : the PDF of the climate response is much more concentrated around the mean value when the negative feedback is added. 


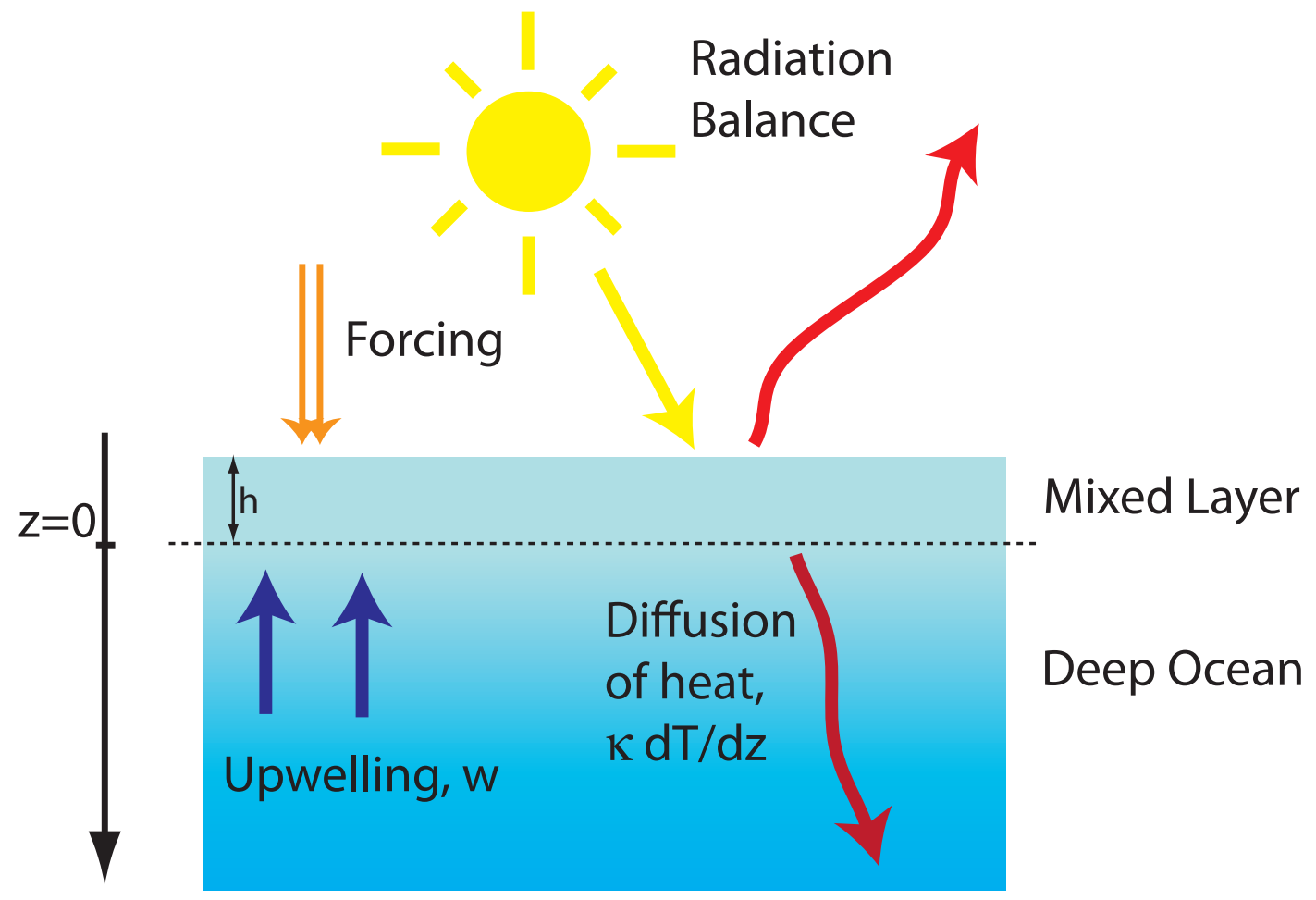

Figure 2: Schematic illustration of the one-dimensional energy balance model. 

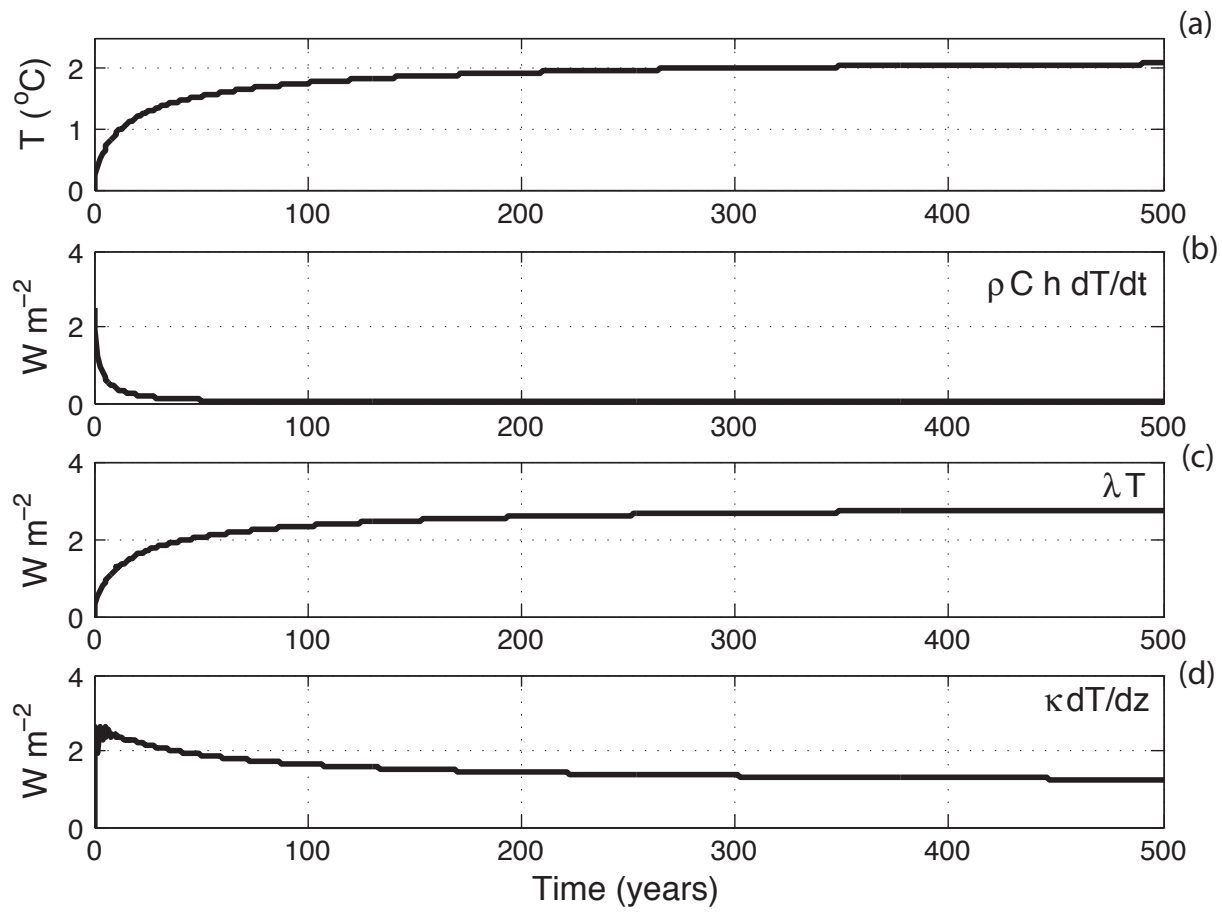

Figure 3: (a) The evolution of the mixed layer temperature in response to a $4 \mathrm{Wm}^{-2}$ step function forcing; (b) to (d) the evolution of three principle terms in the energy balance on the left hand side of (2). The sum of the three terms equals $4 \mathrm{Wm}^{-2}$ at all times. The first term decays quickly to near zero, so that the dominant balance is that between the slow variations of the atmospheric adjustment and the heat driven into the deep ocean. 


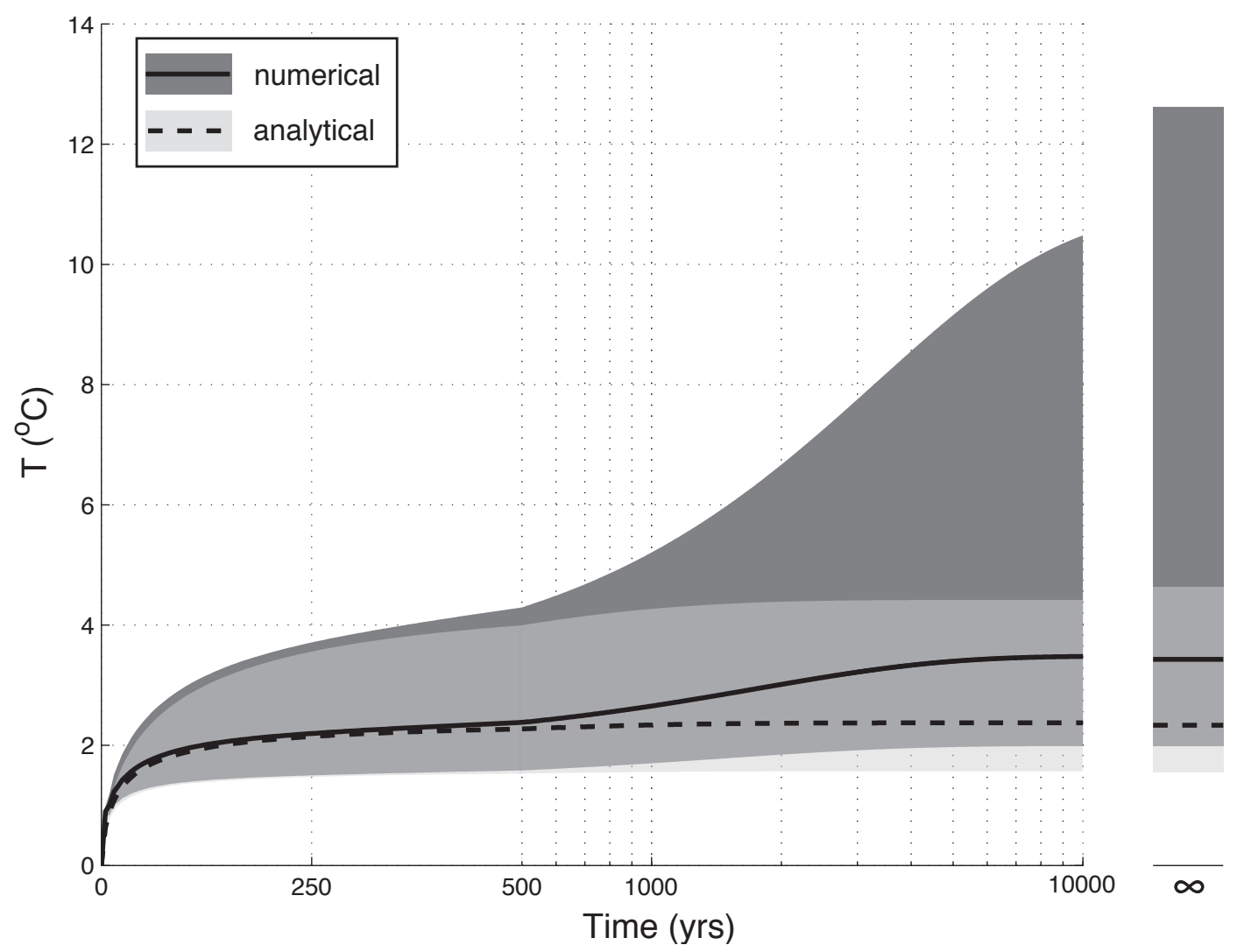

Figure 4: The evolution of the transient climate change to a $4 \mathrm{~W} \mathrm{~m}^{-2}$ step function forcing. The figure shows the comparison between the solutions from the analytical model equations (2) and (3) with seminfinite ocean abyss (light gray), and the numerical model with an ocean floor at $4 \mathrm{~km}$ including transport of warm surface water to the ocean floor (dark gray). See text. The left panel shows the evolution over the first 10,000 years, and the right panel shows the equilibrium response (i.e., the limit of $t \rightarrow \infty$, i.e., the climate sensitivity distribution). The lines show the mean solution based on standard parameters $\left(f_{a}=0.65, f_{o}=-0.15\right)$, and the shading shows $95 \%$ confidence interval based on 1000-member ensemble using $\sigma_{f}=0.13$ with no variation of ocean parameters. The first five hundred years of the climate evolution in the analytical model are nearly identical to the numerical model. 

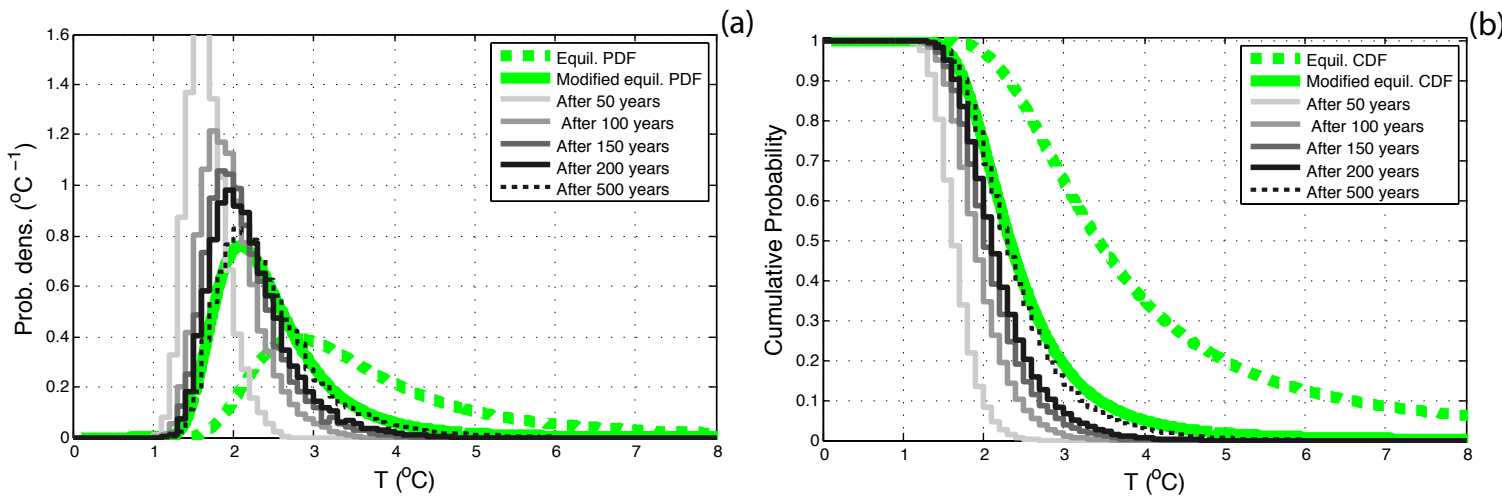

Figure 5: The evolution of the $\mathrm{PDF} h_{T}(T, t)$ (panel a) and (panel b) the cumulative distribution $(\mathrm{CDF}) p_{\text {cum }}\left(T_{c}, t\right)$, the probability that $T \geq T_{c}$ at time $t$. We assume an aquaplanet subject to $\Delta R_{f}=4 \mathrm{Wm}^{-2}$ step function forcing. The histograms were generated from 10,000 Monte Carlo calculations of the analytical model, in which the value of $f_{a}$ was drawn from a normal distribution governed by values in Table 1. Also shown are the equilibrium climate sensitivity probability and cumulative probability distributions calculated from (1), for the atmospheric feedbacks alone (i.e., $f=f_{a}$ ), and also including the effective negative feedback of the ocean heat uptake (i.e., $f=f_{a}+f_{o}$ ), including the same uncertainty in $f_{a}$ as in Figure 1. By about 500 years, the calculated distributions closely match the expected equilibrium distribution of the analytical model (i.e., (1)). 

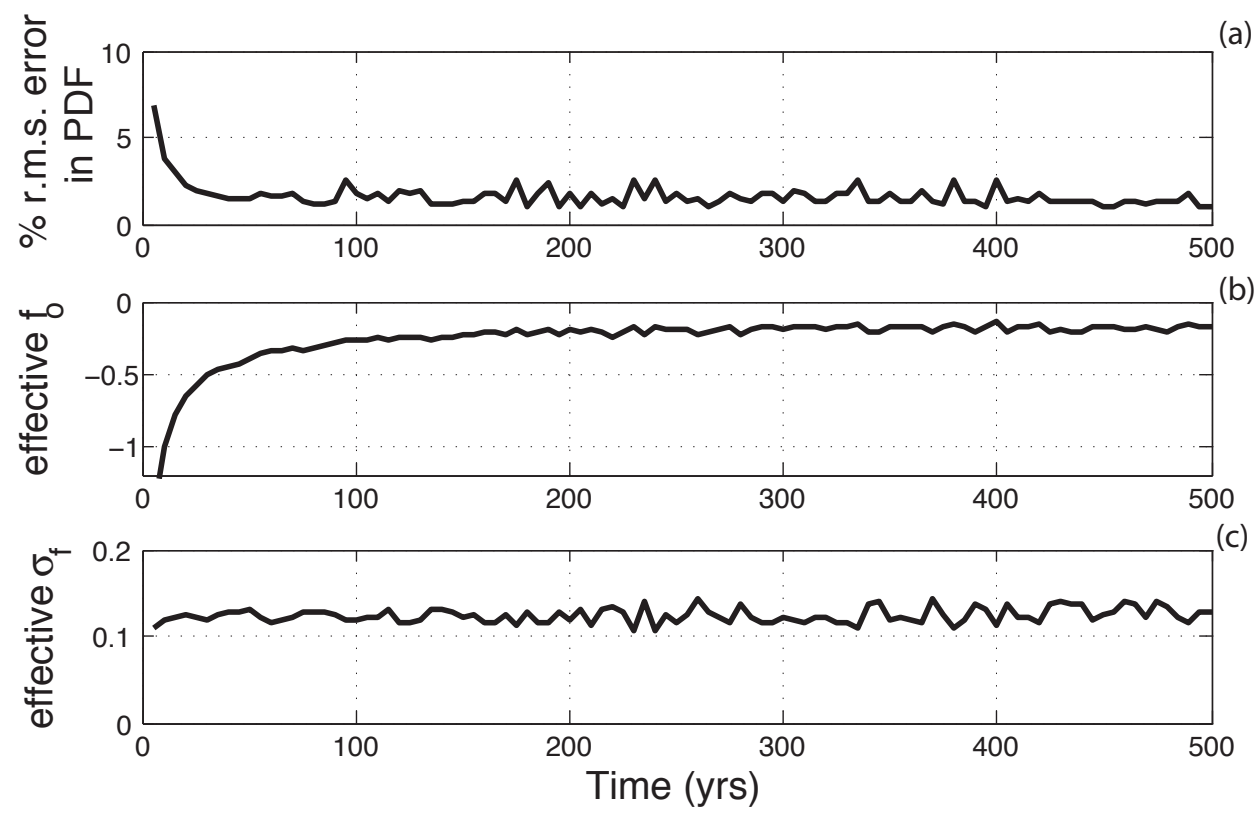

Figure 6: (a) The r.m.s.difference between the evolving PDF and the theoretical distribution for which the $\bar{f}$ and $\sigma_{f}$ in (1) matches the mode of the evolving PDF. Errors are only a few percent after $\sim 20$ years; (b) the effective ocean feedback factor, $\bar{f}_{o} \equiv \bar{f}-\bar{f}_{a}$, found by matching the mode of the evolving PDF with (1). The matched value of $\bar{f}_{o}$ converges to its analytical value of -0.16 after about 100 years; (c) the effective spread in feedback uncertainty, $\sigma_{f}$, found by this procedure. The matched value of $\sigma_{f}$ stays close to 0.13 , the value used in generating the climate PDFs. Panels (a) to (c) shows that, to a high degree of accuracy, the evolving PDF can be well characterized by the theoretical distribution (i.e., (1)) with an effective negative ocean feedback that is strong initially, and diminshes over the medium term to its analytical value. PDFs based on 10,000 Monte Carlo calculations. 
(a)
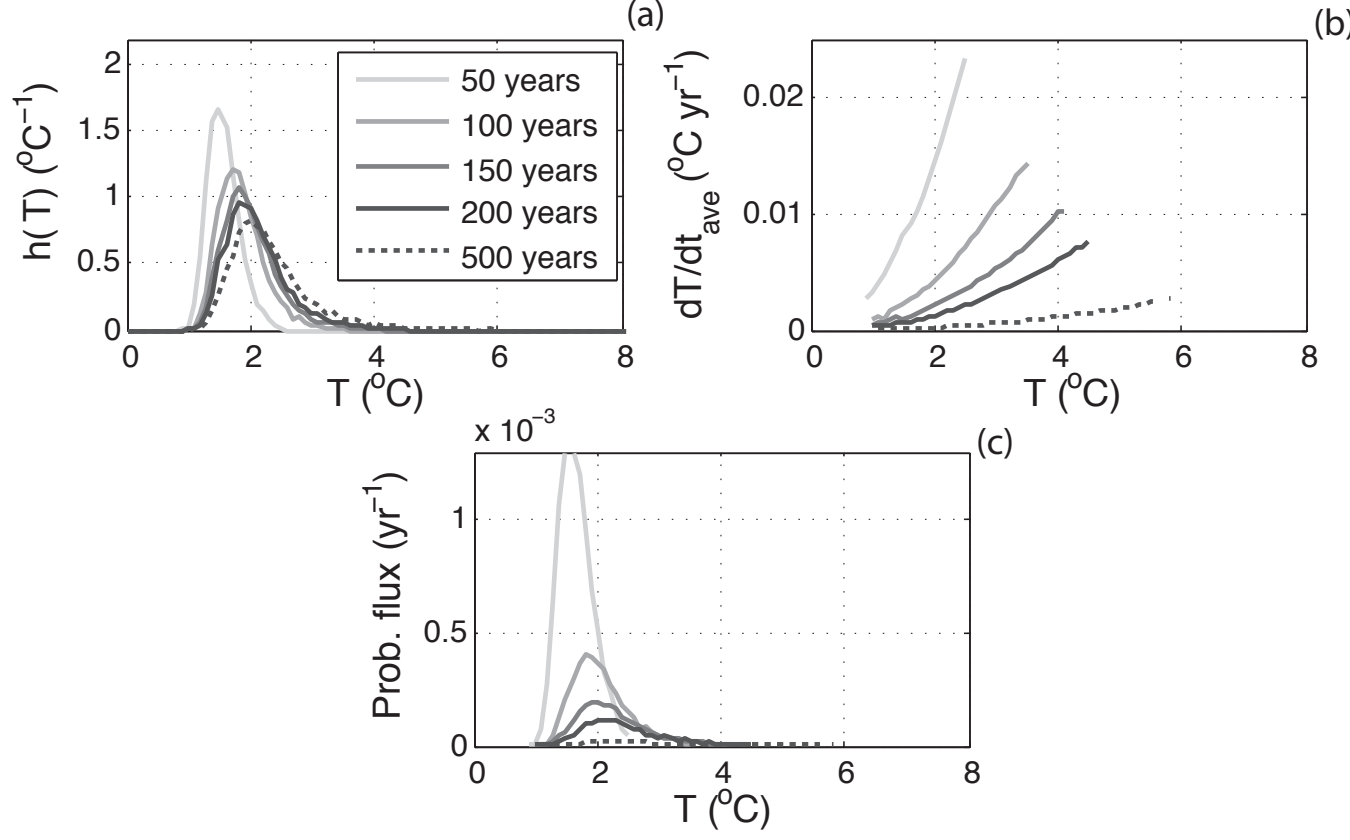

(b)

(c)

Figure 7: The time evolution of the terms in the probability flux equation (i.e., (7)), subject to $\Delta R_{f}=4 \mathrm{Wm}^{-2}$ step function forcing. (a) $h_{T}(T, t)$; (b) the average rate of warming as a function of $T$; (c) the product of (a) and (b), which equals the flux of probability as a function of $T$. This slow flux of probabilities to higher values of $T$ characterizes the growth of the fat tail over time. Based on 10,000 Monte Carlo calculations, asuming uncertainty in $f_{a}$ is governed by a Gaussian distribution based on parameters in Table 1. No uncertainty in ocean parameters is included. 

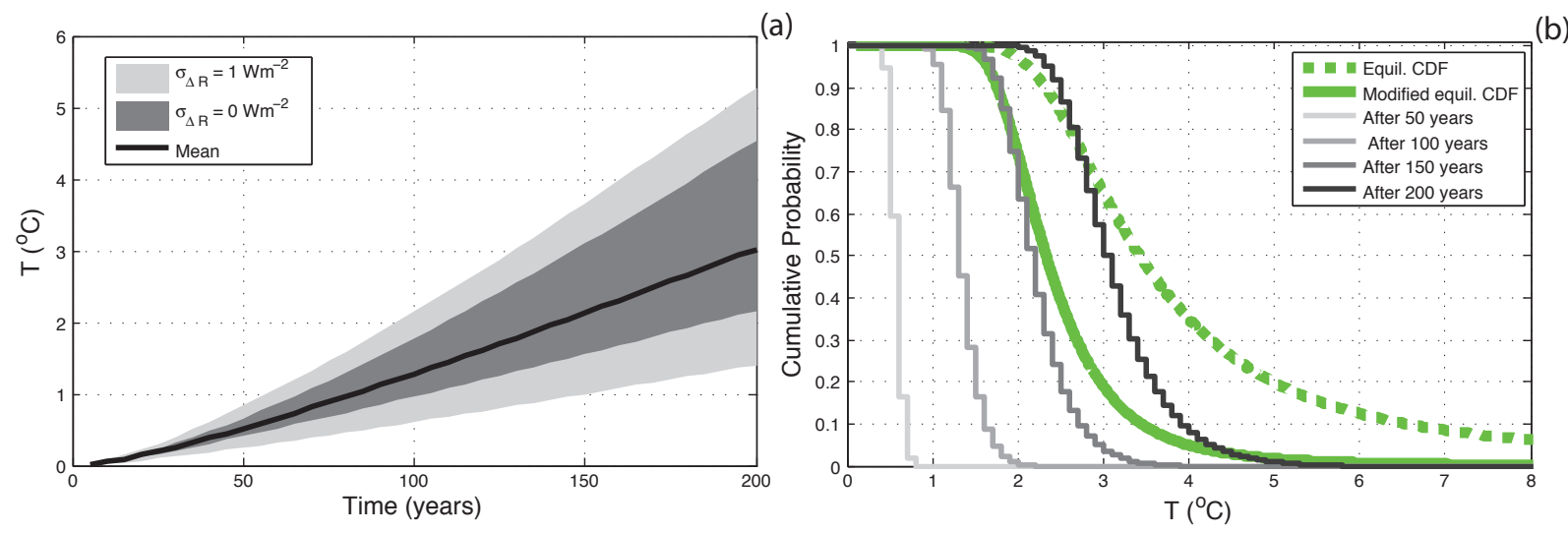

Figure 8: (a) dark shading: $95 \%$ bounds on the time evolution of the surface temperature for a ramp forcing: $\Delta R_{F}(t)=V_{F} t$ where $V_{F}=4 \mathrm{~W} \mathrm{~m}^{-2}(100 \mathrm{yr})^{-1}$; light shading, as for dark shading but including a $25 \%$ standard deviation in uncertainty in $V_{F}$. As explained in the text, after an initial adjustment period, trajectories grow approximately linearly with time. (b) as for (a), but showing the time evolution of the CDF for case with no uncertainty in forcing. The green lines are the same as those in Figure 5b, and are included for comparison. Curves based on 10,000 Monte Carlo calculations, asuming uncertainty in $f_{a}$ is governed by a Gaussian distribution based on parameters in Table 1. 


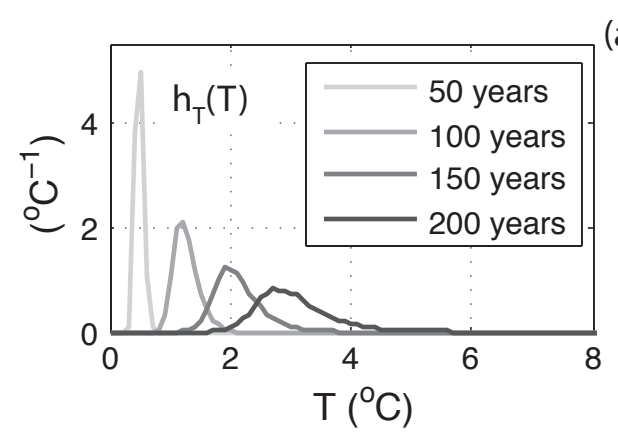

(a)
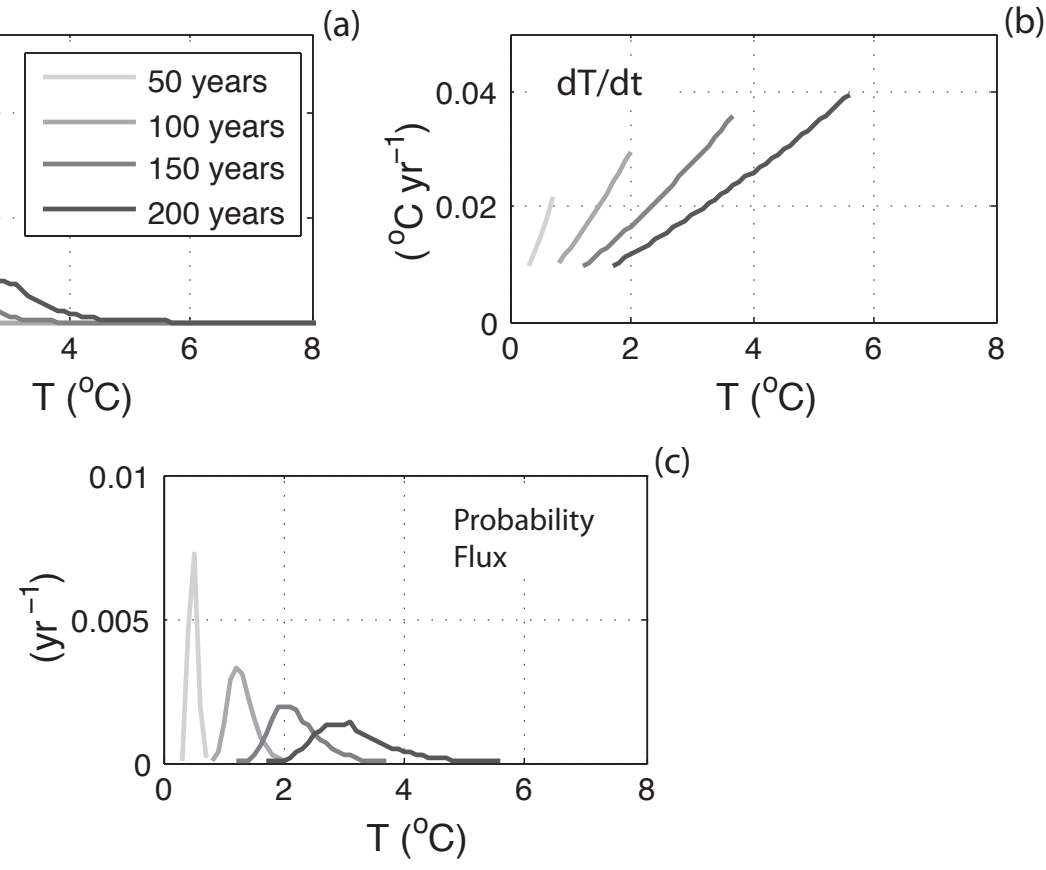

Figure 9: Terms in the probability flux for ramp forcing; same calculations as in Figure 8. The continuous increase in forcing produces a steady flux of probabilities towards higher temperatures, and the distribution broadens with time, as growth at high temperatures is faster than at low temperatures. Compare with Figure 7, but note the different axis scales. 


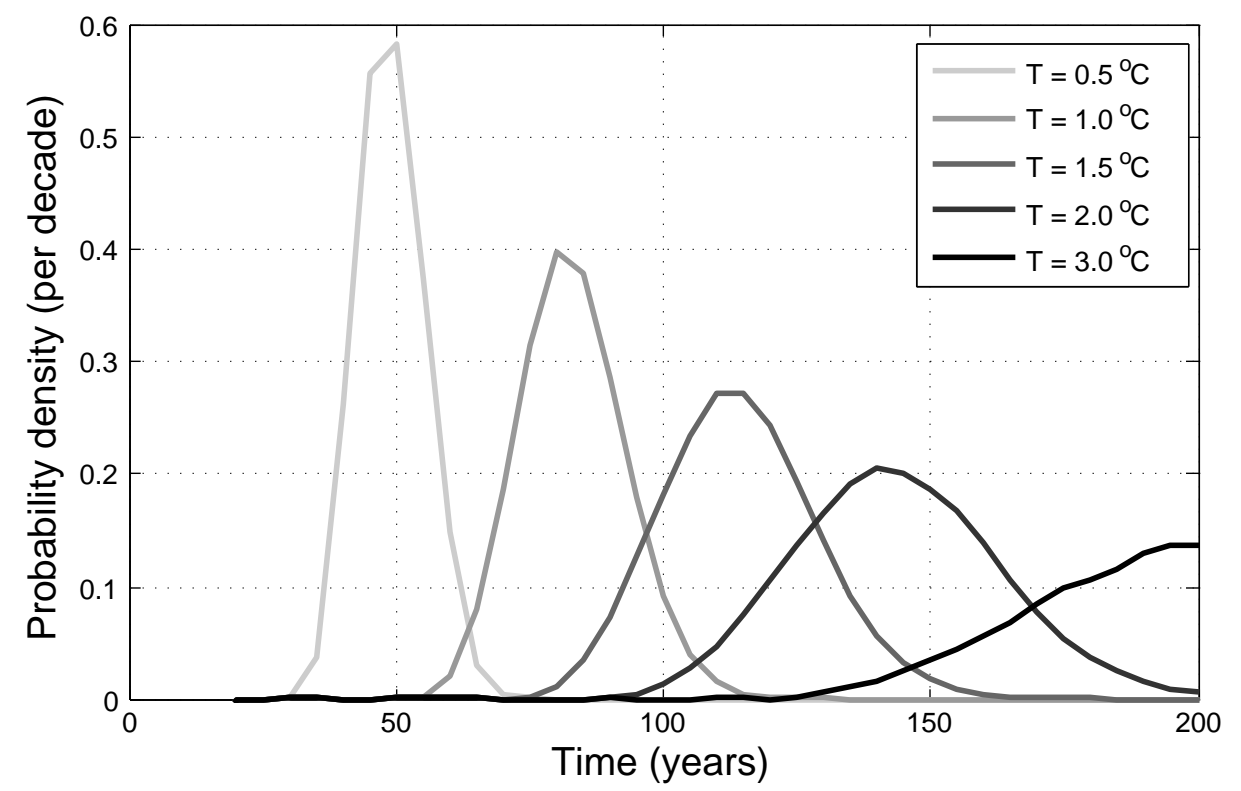

Figure 10: The probability per unit time that the temperature reaches the indicated thresholds, for ramp forcing: $\Delta R_{F}(t)=V_{F} t$ where $V_{F}=4 \mathrm{~W} \mathrm{~m}^{-2}(100 \mathrm{yr})^{-1}$. Here only uncertainties in $f_{a}$ have been considered. While the time to reach lower thresholds can be estimated with some confidence, the time to reach higher thresholds is highly uncertain. 


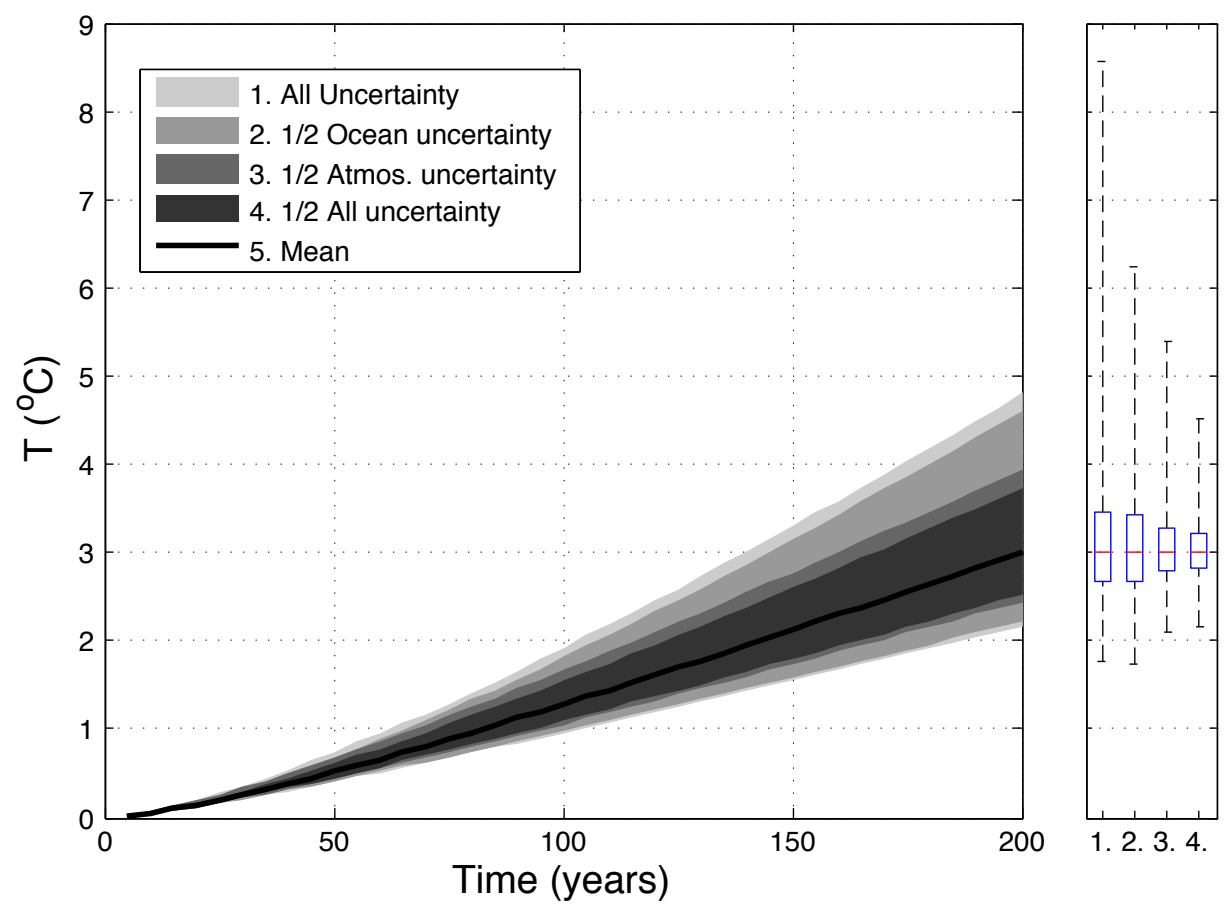

Figure 11: The effect of reducing uncertainty in the model parameters on the time dependent PDF of the climate response (ramp forcing). The shaded regions are $95 \%$ confidence intervals estimated from 10,000 Monte Carlo calculations. The figure shows that it is much more effective to reduce the relative uncertainty in atmospheric feedback factors than to reduce relative uncertainty in ocean heat uptake parameters. The box and whisker plots on the right show the mean, the inter-quartile range, and the extreme limits of the 10,000 calculations. The means and standard deviations of parameters are given in Table 1. 


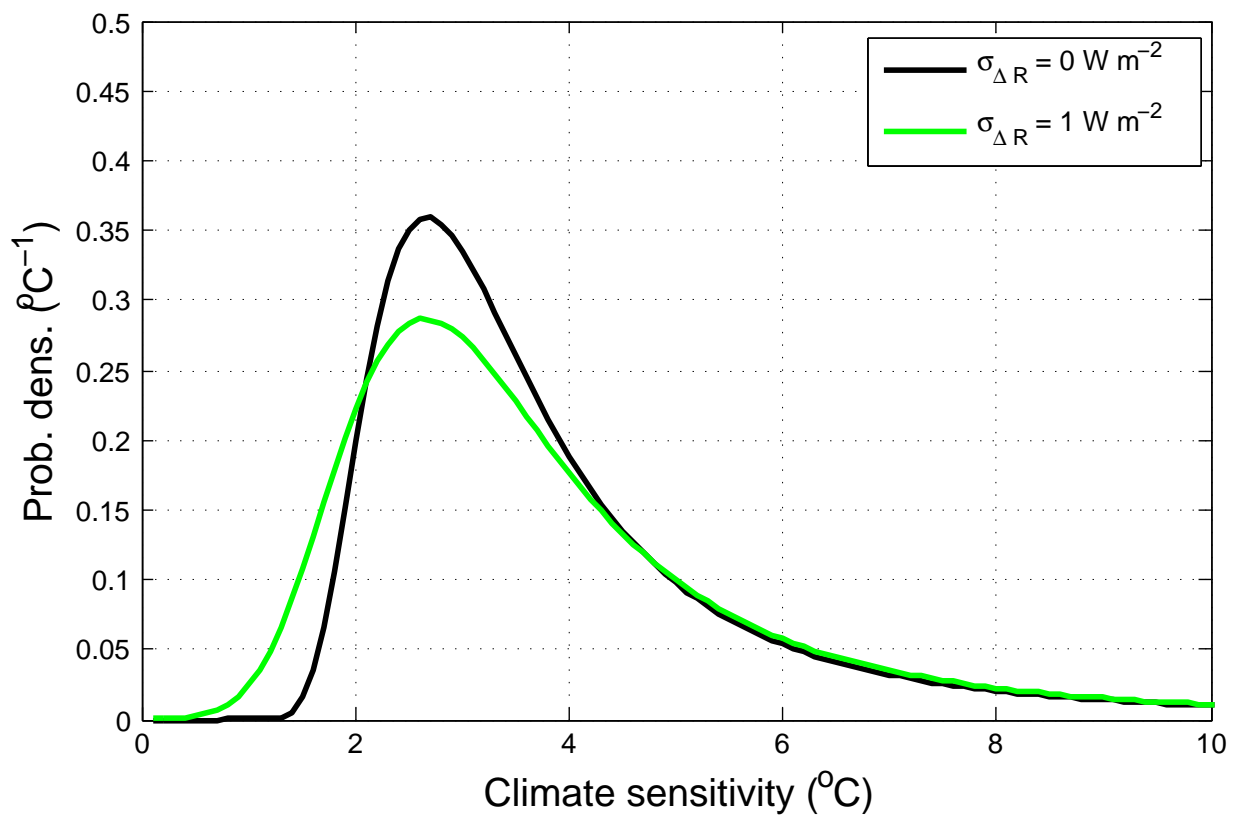

Figure 12: The effect of small symmetric uncertainty in forcing on the equilibrium climate response. The black line shows the PDF of equilibrium climate response for an assumed steady forcing of $\Delta R_{F}=4 \mathrm{~W} \mathrm{~m}^{-2}$. The green line shows the effect of adding a normally-distributed uncertainty in forcing with standard deviation of $1 \mathrm{~W} \mathrm{~m}^{-2}$ : likelihoods are redistributed away from the center of the distribution towards lower climate responses (green line). 


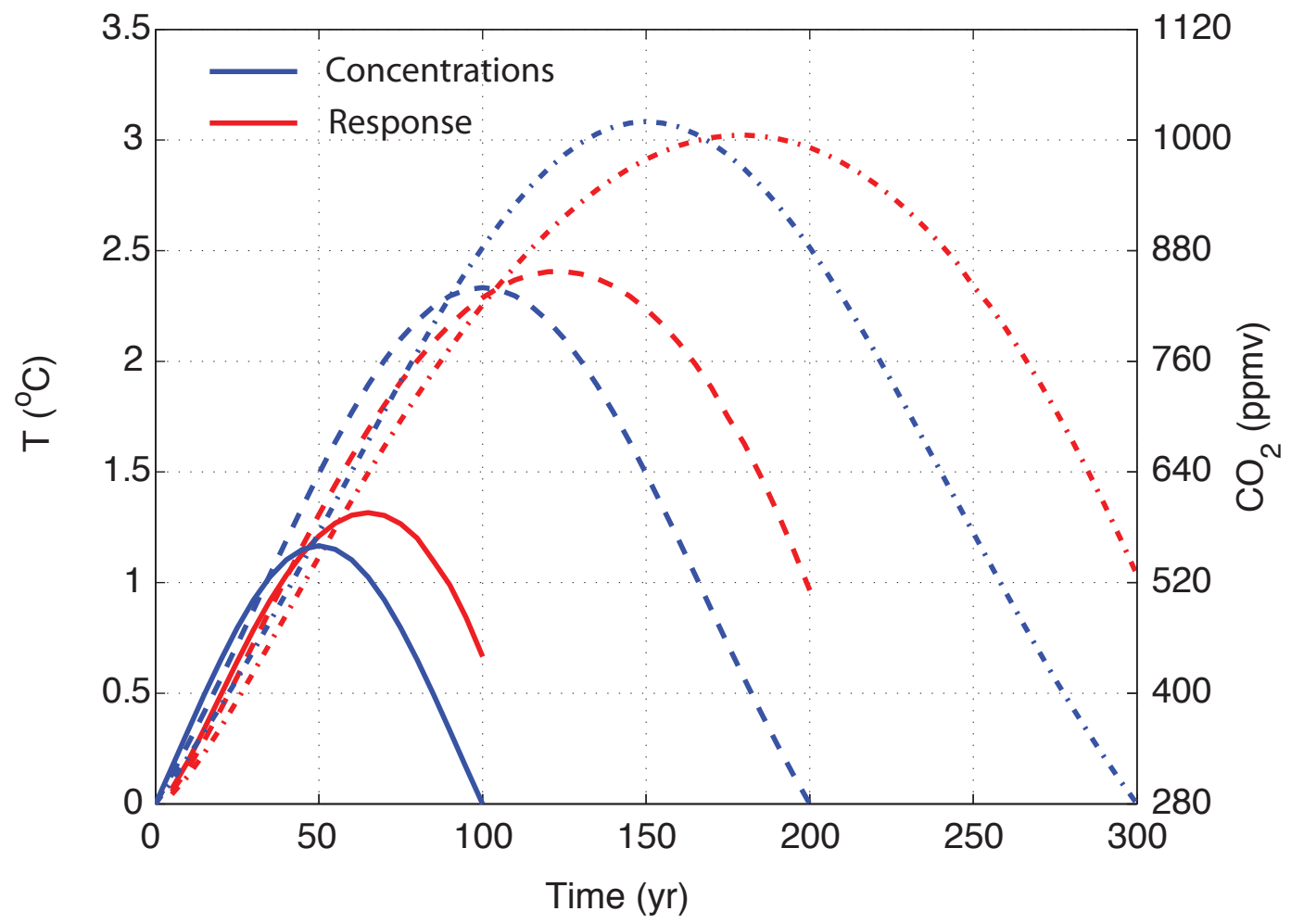

Figure 13: Some examples of concentration trajectories (right axis), and the transient climate change, found from the analytical model (left axis), for the standard set of parameters in Table 1 . 
(a)
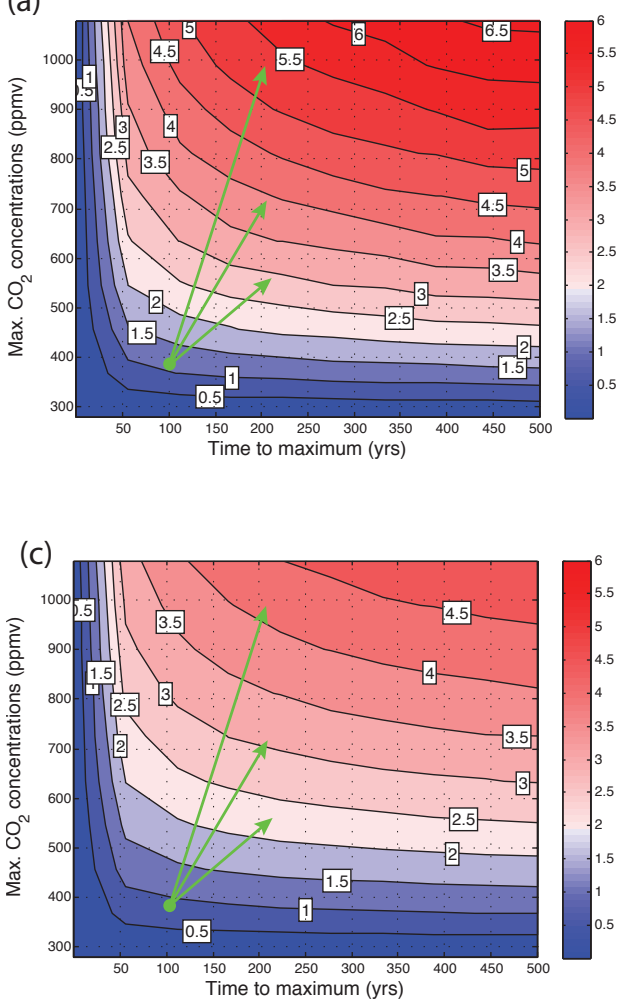

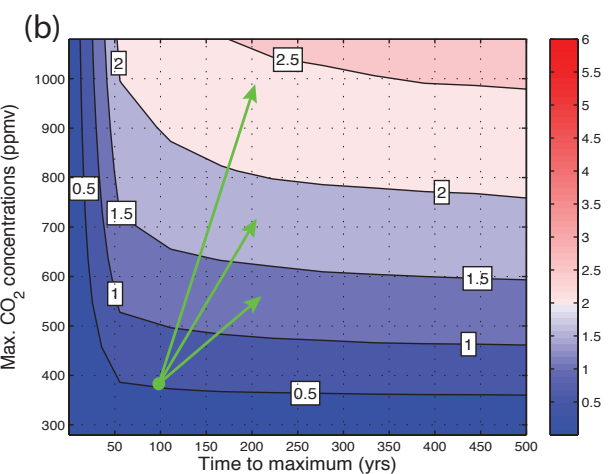

(d)

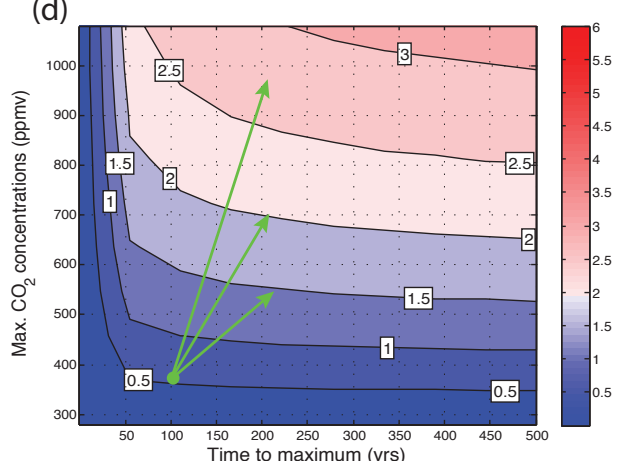

Figure 14: (a) shows $95 \%$ bound on the maximum surface temperature for a range of concentration scenarios, for the assumed uncertainty in the model parameters in Table 1 - that is, for each concentration scenario, the figure shows the temperature change that there is a 1 in 20 chance of exceeding. (b) shows the $5 \%$ lower bound - that is, there is a 19 -in-20 chance of exceeding the given temperature change. Together (a) and (b) bracket the $90 \%$ confidence interval. (c) and (d) show the same calculations as (a) and (b) but for a halving of uncertainty in all model parameters. The green lines show the range of concentrations for the year 2100 considered by IPCC07. See text. Uncertainties in future concentrations are the dominant source of uncertainty in climate change projections. Each panel is based on 10,000 Monte Carlo calculations using the analytical model, for every concentration scenario. 